\title{
Mathematical Modeling of Partial-Porous Circular Cylinders with Water Waves
}

\author{
Min-Su Park ${ }^{1}$ and Weoncheol Koo ${ }^{2}$ \\ ${ }^{1}$ Structural Engineering Research Division, Korea Institute of Civil Engineering and Building Technology, 283 Goyangdae-ro, \\ Ilsanseo-gu, Goyang-si, Gyeonggi-do 411-712, Republic of Korea \\ ${ }^{2}$ Department of Naval Architecture and Ocean Engineering, Inha University, 100 Inha-ro, Nam-gu, Incheon 402-751, Republic of Korea
}

Correspondence should be addressed to Weoncheol Koo; nwavetank@gmail.com

Received 11 September 2014; Accepted 6 November 2014

Academic Editor: Zhen-Lai Han

Copyright (C) 2015 M.-S. Park and W. Koo. This is an open access article distributed under the Creative Commons Attribution License, which permits unrestricted use, distribution, and reproduction in any medium, provided the original work is properly cited.

\begin{abstract}
The interaction of water waves with partially porous-surfaced circular cylinders was investigated. A three-dimensional numerical modeling was developed based on the complete mathematical formulation of the eigenfunction expansion method in the potential flow. Darcy's law was applied to describe the porous boundary. The partial-porous cylinder is composed of a porous-surfaced body near the free surface, and an impermeable-surfaced body with an end-capped rigid bottom below the porous region. The optimal ratio of the porous portion to the impermeable portion can be adopted to design an effective ocean structure with minimal hydrodynamic impact. To scrutinize the hydrodynamic interactions in $N$ partial-porous circular cylinders, the computational fluid domain is divided into three regions: an exterior region, $N$ inner porous body regions, and $N$ regions beneath the body. Wave excitation forces and wave run-up on multibodied partial-porous cylinders are calculated and compared for various porous-portion ratios and wave conditions, all of which significantly influence the hydrodynamic property.
\end{abstract}

\section{Introduction}

Since a circular cylinder has the advantage of a hydrodynamic effect without directivity, an array of vertical circular cylinders is commonly used for coastal and offshore structures. Examples include floating airports, bridges, semisubmersibles, and wave power-conversion systems. In order to design a reliable marine structure that will withstand severe environmental conditions, accurate prediction of hydrodynamic interactions with multibodied structures must be considered. Therefore, research in the area of hydrodynamics has primarily been focused on optimizing systems to avoid significant hydrodynamic impacts. The fundamental idea of dampening the hydrodynamic impact directly on the structure has recently been introduced. One concept involves using porous-surfaced bodies, which can reduce the influence of wave-body interaction through pores on the body surface.

The following is a brief summary of previous research on multibodied circular cylinders with impermeable or porous surfaces. Under the assumption of potential flow and linear wave theory, Spring and Monkmeyer [1] first proposed a semianalytical solution for impermeable cylinders using an eigenfunction expansion approach. This solution was later simplified by Linton and Evans [2] for the case of $N$ bottommounted circular cylinders. Kagemoto and Yue [3] developed another exact solution within the context of linearized theory, showing that a general three-dimensional wave diffraction problem could be solved in terms of algebraically based diffraction characteristics of a single member. This study showed that when the spacing of a multi-cylindrical-column structure is relatively large compared to the incident wavelength, approximation techniques may be used to appraise the hydrodynamic interactions between the cylindrical members. Another popular approach based on the wide-spacing assumption is the modified plane wave method, developed by McIver and Evans [4]. This approach was later applied to a variety of cases by McIver [5], Williams and Demirbilek [6], Williams and Abul-Azm [7], and Williams and Rangappa [8]. Using the eigenfunction method, many researchers studied the hydrodynamic interactions in an array of truncated vertical cylinders ([5, 9-11], etc.); however, the applied models of their studies were all impermeable-surfaced structures. 
For the analysis of $N$ full-body porous-surfaced cylinders, the eigenfunction expansion method can also be employed to describe hydrodynamic interactions in multibodied structures [12-16]. The fluid domain can be divided into $N+1$ regions: a single exterior region and $N$ interior body regions. Wang and Ren [17] first studied the hydrodynamic interactions in a concentric porous cylinder with an impermeable inner surface. Sankarbabu et al. [18] extended this study to a group of concentric, porous, two-surfaced cylinders and calculated the free surface elevations, hydrodynamic forces, and overturning moments analytically. This study showed that the outer porous wall was found to be a critical component in reducing the hydrodynamic forces and wave run-up on the inner rigid wall. Zhao et al. [19] performed theoretical and experimental studies on the interaction of water waves with a truncated porous circular cylinder.

In the present study, with complete mathematical formulations, a three-dimensional potential-flow-based numerical method for an array of surface-piercing, partial-porous cylinders was developed using the eigenfunction expansion method and Darcy's law. The partial-porous cylinder is composed of a porous-surfaced body located near the free surface and a rigid-surfaced body with a rigid end-capped bottom below the porous region. This new type of structure can be used for a variety of marine applications, which require minimum hydrodynamic impact coupled with maximum buoyancy of the submerged module. Using the numerical method, the dynamic response of the new structure due to wave excitation and seismic forces was calculated by Park et al. $[20,21]$ and the reliability evaluation was also carried out by Park et al. [22]. To scrutinize the hydrodynamic interactions in $N$ partial-porous cylinders, the fluid domain is divided into three computational regions: a single exterior region, $N$ inner porous body regions, and $N$ regions beneath the body. The diffracted waves in each potential-flow region can be expressed using the eigenfunction expansion method, under the assumption of linear wave theory. In order to realize the effect of porosity on the cylinder surface, Darcy's law was applied to the porous body boundary condition. In this study, the complete mathematical formulations associated with matching conditions between computational regions were introduced for the diffraction problem of an array of partial-porous cylinders. These are then applied to calculate the wave excitation forces and wave run-up for various conditions. The complete formulations can also be used to evaluate the hydrodynamic properties of any type of circular cylinder, including full-body porous, impermeable, and partial-porous without difficulty.

In order to verify the developed numerical method, the results for an array of truncated full-body porous cylinders are compared with the experimental data of Zhao et al. [19], and the results for full-body impermeable cylinders are compared with the numerical results of the higher-order boundary element method (HOBEM) of Choi et al. [23]. Wave excitation forces and wave run-up on multiple partialporous cylinders are calculated for various wave conditions and strengths of porosity as well as for different ratios of porous wall-length. From the parametric study, the effect of partially porous-surfaced walls on the hydrodynamic properties of the system can be appraised for the optimal design of ocean structures which can withstand the influence of severe marine environments.

\section{Mathematical Formulation}

The boundary value problem for an array of partial-porous circular cylinders can be described using linear potential theory. The fluid in the computational domain is assumed to be an incompressible, inviscid, and irrotational flow. The water particle velocity in the fluid domain can be described using the gradient of the velocity potential $\Phi$. The total velocity potential for a fixed cylinder is composed of the incident wave potential and the resultant scattered potential from the wave-body interaction. An arbitrary array of $N$ partial-porous circular cylinders is situated at a uniform water depth $d$, with the draft $D$. The radius of the $j$ th cylinder is defined as $a_{j}$, and the lengths of the porous and impermeable walls of the respective cylinders are $h$ and $b$, respectively. The global Cartesian coordinate system $(x, y, z)$ is used, and the origin is located at the sea bed with a positive rightward $x$-axis and a vertically upward $z$-axis. The center of each cylinder at $\left(x_{j}, y_{j}\right)$ is taken as the origin of a local polar coordinate system $\left(r_{j}, \theta_{j}\right)$, where $\theta_{j}$ is measured counterclockwise from the positive $x$-axis. The center of the $l$ th cylinder, adjacent to $j$ th cylinder, has polar coordinates $\left(R_{j l}, \alpha_{j l}\right)$. The computational domain is divided into three fluid regions: an interior region of the permeable cylinder ( $r \leq a, d-h \leq z \leq d$, region 1), a region beneath the cylinder $(r \leq a, 0 \leq z \leq d-b-h$, region 2), and an exterior region of cylinders extended to infinity in the horizontal plane ( $r \geq a, 0 \leq z \leq d$, region 3). Both the coordinate system of the $j$ th and $l$ th cylinders and an overview of the computational domain are shown in Figure 1. Basic mathematical parameters and equations of eigenfunction expansion method including boundary conditions in computational domain are also found in Park et al. [20, 21].

The array of surface-piercing partial-porous stationary cylinders is subjected to a train of regular waves of height $H$ and frequency $\omega$, propagating in the positive $x$-direction with an angle $\beta$. The velocity potential of the computational domain can be written as

$$
\Phi(x, y, z, t)=\operatorname{Re}\left[-\left\{\frac{i g H}{(2 \omega)}\right\} \phi(x, y, z) e^{-i \omega t}\right]
$$

where $\operatorname{Re}[\cdot]$ denotes the real part of a complex velocity potential $\Phi$ and $g$ is the gravitational acceleration.

As a governing equation, the Laplace equation is satisfied for the entire fluid domain of the present boundary value problem:

$$
\nabla^{2} \phi=0
$$

For solving the governing equation, the following boundary conditions for the free surface (3), upper bottom, vertical wall, lower bottom of the rigid body $((4) \sim(6))$, flat rigid sea 


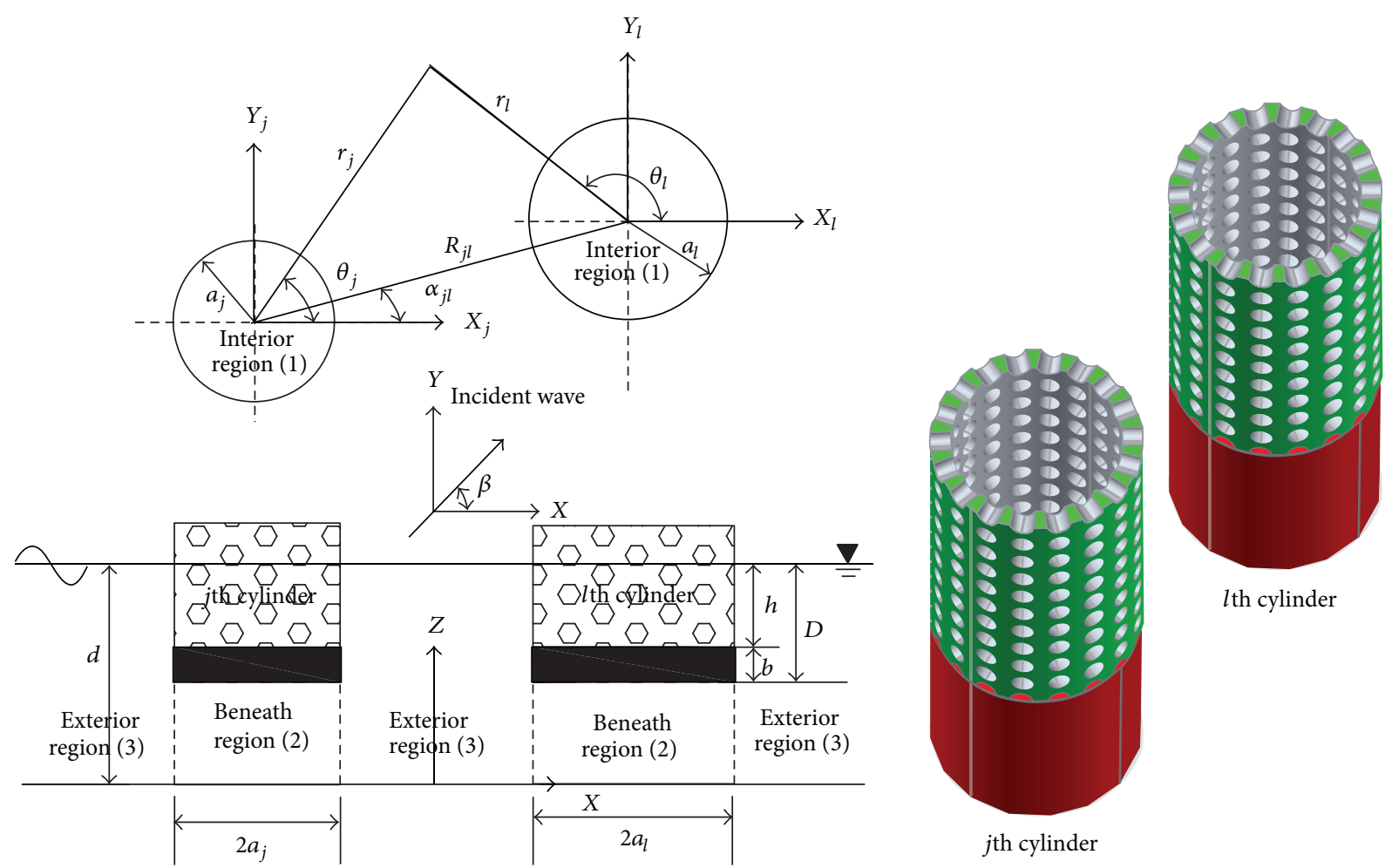

FIGURE 1: Coordinate system for an array of surface-piercing partial-porous cylinders.

bottom (7), and the Sommerfeld radiation boundaries (7) can be given, respectively:

$$
\begin{gathered}
\frac{\partial \phi}{\partial z}-\frac{\omega^{2}}{g} \phi=0 \quad \text { on } z=d, \\
\frac{\partial \phi}{\partial z}=0 \quad \text { on } z=d-h, 0 \leq r \leq a, \\
\frac{\partial \phi}{\partial r}=0 \quad \text { on } r=a,(d-h-b) \leq z \leq(d-h), \\
\frac{\partial \phi}{\partial z}=0 \quad \text { on } z=d-h-b, 0 \leq r \leq a, \\
\frac{\partial \phi}{\partial z}=0 \quad \text { on } z=0, \\
\lim _{r \rightarrow \infty} \sqrt{r}\left[\frac{\partial}{\partial r}\left(\phi_{3}-\phi_{\text {in }}\right)-i k\left(\phi_{3}-\phi_{\text {in }}\right)\right]=0,
\end{gathered}
$$

where $k$ is the incident wave number related to the wave frequency through the dispersion relation $\omega^{2}=g k \tanh k d$ and $d$ is the water depth. $\phi_{3}$ and $\phi_{\text {in }}$ denote the total velocity potential in region 3 and the incident wave potential, respectively.

The incident wave potential in the $j$ th local polar coordinate system can be expressed as

$$
\phi_{\text {in }}^{j}=\frac{\cosh k z}{\cosh k d} T_{j} e^{i k r_{j} \cos \left(\theta_{j}-\beta\right)},
$$

where $T_{j}=e^{i k\left(x_{j} \cos \beta+y_{j} \sin \beta\right)}$ is a phase factor associated with the cylinder $j$ from the global origin. Equation (9) can be reformulated using the Jacobi-Anger expansion of Bessel functions as follows:

$$
\phi_{\text {in }}^{j}=\frac{\cosh k z}{\cosh k d} T_{j} \sum_{n \rightarrow-\infty}^{\infty} J_{n}\left(k r_{j}\right) e^{\operatorname{in}\left(\pi / 2+\theta_{j}-\beta\right)},
$$

where $J_{n}$ denotes the Bessel function of the first kind of order $n$.

The scattered wave potential from the cylinder $j$ can be described in a generalized form:

$$
\begin{aligned}
\phi_{s}^{j}= & \frac{\cosh k z}{\cosh k d} \sum_{n \rightarrow-\infty}^{\infty} C_{n}^{j} \frac{H_{n}\left(k r_{j}\right)}{H_{n}^{\prime}\left(k a_{j}\right)} e^{\mathrm{in} \theta_{j}} \\
& +\sum_{q=1}^{\infty} \frac{\cos k_{q} z}{\cos k_{q} d_{n \rightarrow-\infty}} \sum_{n \rightarrow}^{\infty} \frac{K_{n}^{j}\left(k_{q} r_{j}\right)}{K_{n}^{\prime}\left(k_{q} a_{j}\right)} e^{\mathrm{in} \theta_{j}},
\end{aligned}
$$

where $C_{n}^{j}$ and $C_{n q}^{j}$ are unknown complex potential coefficients. $H_{n}$ and $K_{n}$ are a Hankel function of the first kind and a modified Bessel function of the second kind of order $n$. $H_{n}^{\prime}$ and $K_{n}^{\prime}$ are the first derivatives of the Hankel and the modified Bessel functions, respectively. The scattered wave potential in (11) is composed of the propagating wave (first term) and the local (evanescent) wave mode (second term). The evanescent wave mode represents a standing wave system near the body and exists only near the body. The wave numbers $\left(k_{q}\right.$, positive real roots) associated with such local waves satisfy the dispersion relation $\omega^{2}=-g k_{q} \tan \left(k_{q} d\right)$. 
When combining the incident wave potential (equation (10)) with the scattered potential (equation (11)), the total potential in the exterior region (region 3 ) can be formulated as

$$
\begin{aligned}
\phi_{3}^{j}=\phi_{\text {in }}^{j} & +\sum_{j=1}^{N} \phi_{s}^{j}=\frac{\cosh k z}{\cosh k d} T_{j} \sum_{n=-\infty}^{\infty} J_{n}\left(k r_{j}\right) e^{\operatorname{in}\left(\pi / 2+\theta_{j}-\beta\right)} \\
+ & \sum_{j=1}^{N}\left\{\frac{\cosh k z}{\cosh k d} \sum_{n \rightarrow-\infty}^{\infty} C_{n}^{j} \frac{H_{n}\left(k r_{j}\right)}{H_{n}^{\prime}\left(k a_{j}\right)} e^{\operatorname{in} \theta_{j}}\right. \\
& \left.+\sum_{q=1}^{\infty} \frac{\cos k_{q} z}{\cos k_{q} d} \sum_{n \rightarrow-\infty}^{\infty} C_{n q}^{j} \frac{K_{n}\left(k_{q} r_{j}\right)}{K_{n}^{\prime}\left(k_{q} a_{j}\right)} e^{\operatorname{in} \theta_{j}}\right\}
\end{aligned}
$$

Using Graf's addition theorem for Bessel functions [24], the scattered potential in the exterior region with respect to any local cylinder's coordinate system can be expressed as

$$
\begin{aligned}
& \phi_{3}^{j}\left(r_{j}, \theta_{j}\right) \\
& =\sum_{n=-\infty}^{\infty}\left[\left\{T_{j} J_{n}\left(k r_{j}\right) e^{i n(\pi / 2-\beta)}+C_{n}^{j} \frac{H_{n}\left(k r_{j}\right)}{H_{n}^{\prime}\left(k a_{j}\right)}\right.\right. \\
& \left.+\sum_{\substack{l=1 \\
l \neq j}}^{N} \sum_{m=-\infty}^{\infty} C_{m}^{l} \frac{J_{n}\left(k r_{j}\right)}{H_{n}^{\prime}\left(k a_{j}\right)} H_{m-n}\left(k R_{l j}\right) e^{i(m-n) \alpha_{l j}}\right\} \\
& \times \frac{\cosh k z}{\cosh k d} \\
& +\sum_{q=1}^{\infty}\left\{C_{n q}^{j} \frac{K_{n}\left(k_{q} r_{j}\right)}{K_{n}^{\prime}\left(k_{q} a_{j}\right)}\right. \\
& +\sum_{\substack{l=1 \\
l \neq j}}^{N} \sum_{m=-\infty}^{\infty} C_{m q}^{l} \frac{I_{n}\left(k_{q} r_{j}\right)}{K_{n}^{\prime}\left(k_{q} a_{j}\right)} \\
& \left.\times K_{m-n}\left(k_{q} R_{l j}\right) e^{i(m-n) \alpha_{l j}} e^{-i m \pi}\right\} \\
& \left.\times \frac{\cos k_{q} z}{\cos k_{q} d}\right] \times e^{\operatorname{in} \theta_{j}}
\end{aligned}
$$

where the equation is valid only if $r_{j}<R_{l j}$ for all $j . I_{n}$ is a modified Bessel function of the first kind of order $n$. The terms in the right-hand side of (13) represent the incident wave upon the $j$ th cylinder, the scattered waves by the $j$ th cylinder, and the rescattered waves by the adjacent cylinders $l$, respectively.
The wave potential of the $j$ th cylinder in the interior region (region 1), which satisfies the linearized free surface and body boundary conditions, can be expressed by the following eigenfunction expansions:

$$
\begin{aligned}
\phi_{1}^{j}=\sum_{n=-\infty}^{\infty}\left[A_{n}^{j} \frac{J_{n}\left(k_{0} r_{j}\right)}{J_{n}\left(k_{0} a_{j}\right)} \frac{\cosh \left\{k_{0}(z-d+h)\right\}}{\cosh \left(k_{0} h\right)}\right. \\
\left.+\sum_{q=1}^{\infty} A_{n q}^{j} \frac{I_{n}\left(k_{0 q} r_{j}\right)}{I_{n}\left(k_{0 q} a_{j}\right)} \frac{\cos \left\{k_{0 q}(z-d+h)\right\}}{\cos \left(k_{0 q} h\right)}\right] e^{\text {in } \theta_{j}} \\
\text { on } d-h \leq z \leq d,
\end{aligned}
$$

where a new wave number $k_{0}$ is introduced from the dispersion relation $\omega^{2}=g k_{0} \tanh k_{0} h . A_{n}^{j}$ and $A_{n q}^{j}$ are unknown complex coefficients, and $h$ is the vertical length of the porous-surfaced body. $k_{0 q}$ (positive real roots) is related to the evanescent waves, which satisfy the dispersion relation $\omega^{2}=$ $-g k_{0 q} \tan \left(k_{0 q} h\right)$.

The wave potential beneath the $j$ th cylinder (region 2), which satisfies the rigid cylinder bottom (equation (6)) and flat rigid sea bottom boundary conditions (equation (7)), can also be given in the form of eigenfunction expansions:

$$
\begin{array}{r}
\phi_{2}^{j}=\sum_{n=-\infty}^{\infty}\left[\frac{B_{n, 0}^{j}}{2}\left(\frac{r_{j}}{a_{j}}\right)^{n}+\sum_{q=1}^{\infty} B_{n, q}^{j} \frac{I_{n}\left(b_{0} r_{j}\right)}{I_{n}\left(b_{0} a_{j}\right)} \cos \left(b_{0} z\right)\right] e^{\text {in } \theta_{j}} \\
\text { on } 0 \leq z \leq d-h-b,
\end{array}
$$

where $B_{n, 0}^{j}$ and $B_{n, q}^{j}$ are unknown complex coefficients and $b_{0}=q \pi /(d-b-h)$. In addition to applying the body boundary conditions associated with the free surface conditions, the present boundary value problem must satisfy the matching conditions at the interface between the regions, which are given by

$$
\begin{gathered}
\frac{\partial \phi_{1}^{j}}{\partial r}=\frac{\partial \phi_{3}^{j}}{\partial r} \quad \text { on } r_{j}=a_{j}, d-h \leq z \leq d, \\
\phi_{2}^{j}=\phi_{3}^{j} \quad \text { on } r_{j}=a_{j}, 0 \leq z \leq d-h-b, \\
\frac{\partial \phi_{2}^{j}}{\partial r}=\frac{\partial \phi_{3}^{j}}{\partial r} \quad \text { on } r_{j}=a_{j}, 0 \leq z \leq d-h-b .
\end{gathered}
$$

In order to account for the porous-body boundary condition, the circular cylinder near the free surface has a thin-surfaced wall containing small pores, which allow water particles to pass through, where Darcy's law $\left(Q=k_{d} A\left(P_{3}-\right.\right.$ $\left.P_{1}\right) / L$ ) is assumed to be obeyed. The total discharge, $Q$ (the volumetric flow through the filter), is equal to the product of the proportionality constant $\left(k_{d}\right)$, the cross-sectional area $(A)$, and the pressure drop $\left(P_{3}-P_{1}\right)$ per unit filter length $(L)$. Hence, the fluid velocity is linearly proportional to the pressure difference between the interior and exterior walls of the cylinder. The hydrodynamic pressure $p(x, y, z, t)=$ 
$\operatorname{Re}\left[P(x, y) e^{-i \omega t} \cosh k_{0}(z-d+h) / \cosh k_{0} h\right]$ can then be obtained at any point in the fluid domain using the linearized Bernoulli equation, $P(x, y)=i \rho \omega \phi(x, y)$, where $\rho$ is the water density. Therefore, the porous-body boundary condition can be given as [13]

$$
\frac{\partial \phi_{1}^{j}}{\partial r}=\frac{\gamma}{\mu} \rho i \omega\left[\phi_{1}^{j}-\phi_{3}^{j}\right] \quad \text { on } r_{j}=a_{j}, j=1,2,3, \ldots, N \text {, }
$$

where $\mu$ is the dynamic viscosity coefficient and $\gamma$ is a material constant with a unit of length. Subsequently, the porous boundary condition on the cylinder is also described with a dimensionless parameter, $G$ :

$$
\frac{\partial \phi_{1}^{j}}{\partial r}=i k_{0} G\left[\phi_{1}^{j}-\phi_{3}^{j}\right]
$$

where $G=\rho \omega \gamma / \mu k_{0}$.

Substituting (13) and (14) and using the orthogonality of depth from $z=d-h$ to $d$, (18) can be rewritten as follows.

(i) In the case of $k$ (propagating wave number),

$$
\begin{aligned}
& \sum_{n=-\infty}^{\infty} A_{n}^{j}\left\{1+\frac{i J_{n}^{\prime}\left(k_{0} a_{j}\right)}{J_{n}\left(k_{0} a_{j}\right) G}\right\} \\
& \quad \times \int_{d-h}^{d}\left\{\frac{\cosh \left\{k_{0}(z-d+h)\right\}}{\cosh \left(k_{0} h\right)}\right\}^{2} d z \\
& =\sum_{n=-\infty}^{\infty}\left[T_{j} J_{n}\left(k a_{j}\right) e^{\operatorname{in}(\pi / 2-\beta)}+C_{n}^{j} \frac{H_{n}\left(k a_{j}\right)}{H_{n}^{\prime}\left(k a_{j}\right)}\right. \\
& \quad+\sum_{l=1}^{N} \sum_{m=-\infty}^{m=\infty} C_{m}^{l} \frac{J_{n}\left(k a_{j}\right)}{H_{m}^{\prime}\left(k a_{l}\right)} H_{m-n}\left(k R_{l j}\right) e^{i(m-n) \alpha_{l j}} \\
& \quad \times \int_{d-h}^{d}\left\{\frac{\cosh k z}{\cosh k d} \frac{\cosh \left\{k_{0}(z-d+h)\right\}}{\cosh \left(k_{0} h\right)}\right\} d z .
\end{aligned}
$$

(ii) In the case of $k_{q}$ (evanescent wave number),

$$
\begin{aligned}
& \sum_{n=-\infty}^{\infty} A_{n}^{j}\left\{1+\frac{i I_{n}^{\prime}\left(k_{0 q} a_{j}\right)}{I_{n}\left(k_{0 q} a_{j}\right) G}\right\} \\
& \quad \times \int_{d-h}^{d}\left\{\frac{\cos \left\{k_{0 q}(z-d+h)\right\}}{\cos \left(k_{0 q} h\right)}\right\}^{2} d z
\end{aligned}
$$

$$
\begin{gathered}
=\sum_{n=-\infty}^{\infty}\left[\sum _ { q = 1 } ^ { \infty } \left\{C_{n q}^{j} \frac{K_{n}\left(k_{q} a_{j}\right)}{K_{n}^{\prime}\left(k_{q} a_{j}\right)}\right.\right. \\
+\sum_{\substack{l=1 \\
l \neq j}}^{N} \sum_{m=-\infty}^{\infty} C_{m q}^{l} \frac{I_{n}\left(k_{q} a_{j}\right)}{K_{m}^{\prime}\left(k_{q} a_{l}\right)} \\
\left.\times K_{m-n}\left(k_{q} R_{l j}\right) e^{i(m-n) \alpha_{l j}} e^{-i m \pi}\right\} \\
\left.\times \int_{d-h}^{d}\left\{\frac{\cos k_{q} z \cos \left\{k_{0 q}(z-d+h)\right\}}{\cos k_{q} d}\right\} d z\right] .
\end{gathered}
$$

For the second matching condition between regions 2 and 3 in (16), the equation can be reformulated with the orthogonality of depth from $z=0$ to $d-h-b$ as follows.

(i) In the case of $k$ (propagating wave number),

$$
\begin{aligned}
& \sum_{n=-\infty}^{\infty} \frac{B_{n, 0}^{j}}{2} \int_{0}^{d-h-b} \cos \left(b_{0} z\right) d z+\sum_{q=1}^{\infty} B_{n, q}^{j} \int_{0}^{d-h-b}\left\{\cos \left(b_{0} z\right)\right\}^{2} d z \\
& =\sum_{n=-\infty}^{\infty}\left[T_{j} J_{n}\left(k a_{j}\right) e^{\operatorname{in}(\pi / 2-\beta)}+C_{n}^{j} \frac{H_{n}\left(k a_{j}\right)}{H_{n}^{\prime}\left(k a_{j}\right)}\right. \\
& +\sum_{l=1}^{N} \sum_{m=-\infty}^{\infty} C_{m}^{l} \frac{J_{n}\left(k a_{j}\right)}{H_{n}^{\prime}\left(k a_{l}\right)} \\
& \left.\quad \times H_{m-n}\left(k R_{l j}\right) e^{i(m-n) \alpha_{l j}}\right] \\
& \quad \times \int_{0}^{d-h-b} \frac{\cosh k z}{\cosh k d} \cos \left(b_{0} z\right) d z .
\end{aligned}
$$

(ii) In the case of $k_{q}$ (evanescent wave number),

$$
\begin{gathered}
\sum_{n=-\infty}^{\infty} \frac{B_{n, 0}^{j}}{2} \int_{0}^{d-h-b} \cos \left(b_{0} z\right) d z+\sum_{q=1}^{\infty} B_{n, q}^{j} \int_{0}^{d-h-b}\left\{\cos \left(b_{0} z\right)\right\}^{2} d z \\
=\sum_{n=-\infty}^{\infty}\left[\sum _ { q = 1 } ^ { \infty } \left\{C_{n q}^{j} \frac{K_{n}\left(k_{q} a_{j}\right)}{K_{n}^{\prime}\left(k_{q} a_{j}\right)}\right.\right. \\
+\sum_{\substack{l=1 \\
l \neq j}}^{N} \sum_{m=-\infty}^{\infty} C_{m q}^{l} \frac{I_{n}\left(k_{q} a_{j}\right)}{K_{n}^{\prime}\left(k_{q} a_{l}\right)}
\end{gathered}
$$




$$
\left.\begin{array}{c}
\times K_{m-n}\left(k_{q} R_{l j}\right) e^{i(m-n) \alpha_{l j}} e^{-i m \pi} \\
\left.\times \int_{0}^{d-h-b} \frac{\cos k_{q} z}{\cos k_{q} d} \cos \left(b_{0} z\right) d z\right]
\end{array}\right]
$$

Applying the orthogonal property to the first and third matching conditions in (16), with respect to $z$ over the region of validity, the following equation can be obtained.

In the case of $k$ (propagating wave number),

$$
\begin{aligned}
\int_{0}^{d} \frac{\partial \phi_{3}^{j}}{\partial r}\left\{\frac{\cosh k z}{\cos k d}\right\} d z= & \int_{0}^{d-h-b} \frac{\partial \phi_{2}^{j}}{\partial r} \frac{\cosh k z}{\cos k d} d z \\
& +\int_{d-h-b}^{d-h} \frac{\partial \phi_{3}^{j}}{\partial r} \frac{\cosh k z}{\cos k d} d z \\
& +\int_{d-h}^{d} \frac{\partial \phi_{1}^{j}}{\partial r} \frac{\cosh k z}{\cos k d} d z
\end{aligned}
$$

By applying (19) and (21) to (23), the key equation for the present boundary value problem can then be rearranged in the form of an infinite system.

In the case of $k$ (propagating wave number),

$$
\begin{aligned}
& \left\{T_{j} e^{i n(\pi / 2-\beta)} J_{n}^{\prime}\left(k a_{j}\right) k+C_{n}^{j} k\right. \\
& +\sum_{\substack{l=1 \\
l \neq j}}^{N} \sum_{m=-\infty}^{\infty} C_{m}^{l} \frac{J_{n}^{\prime}\left(k a_{j}\right)}{H_{n}^{\prime}\left(k a_{l}\right)} \\
& \left.\times k H_{m-n}\left(k R_{l j}\right) e^{i(m-n) \alpha_{l j}}\right\} \\
& \times \int_{0}^{d}\left\{\frac{\cosh (k z)}{\cosh (k d)}\right\}^{2} d z \\
& =\frac{|n|}{2 a_{j}} \frac{2}{d-h-b} \\
& \times\left\{\left[T_{j} J_{n}\left(k a_{j}\right) e^{i n(\pi / 2-\beta)}+C_{n}^{j} \frac{H_{n}\left(k a_{j}\right)}{H_{n}^{\prime}\left(k a_{j}\right)}\right.\right.
\end{aligned}
$$

$$
\begin{aligned}
& +\sum_{\substack{l=1 \\
l \neq j}}^{N} \sum_{m=-\infty}^{\infty} C_{m}^{l} \frac{J_{n}\left(k a_{j}\right)}{H_{m}^{\prime}\left(k a_{l}\right)} \\
& \left.\left.\times H_{m-n}\left(k R_{l j}\right) e^{i(m-n) \alpha_{l j}}\right]\right\} \\
& \times \int_{0}^{d-h-b} \frac{\cosh (k z)}{\cosh (k d)} d z \int_{0}^{d-h-b} \frac{\cosh (k z)}{\cosh (k d)} d z \\
& +\sum_{q=1}^{\infty} \frac{I_{n}^{\prime}\left(b_{0} a_{j}\right) b_{0}}{I_{n}\left(b_{0} a_{j}\right)} \\
& \times\left\{\left[T_{j} J_{n}\left(k a_{j}\right) e^{\mathrm{in}(\pi / 2-\beta)}+C_{n}^{j} \frac{H_{n}\left(k a_{j}\right)}{H_{n}^{\prime}\left(k a_{j}\right)}\right.\right. \\
& +\sum_{\substack{l=1 \\
l \neq j}}^{N} \sum_{m=-\infty}^{\infty} C_{m}^{l} \frac{J_{n}\left(k a_{j}\right)}{H_{m}^{\prime}\left(k a_{l}\right)} \\
& \left.\left.\times H_{m-n}\left(k R_{l j}\right) e^{i(m-n) \alpha_{l j}}\right]\right\} \\
& \times \int_{0}^{d-h-b}\left\{\frac{\cosh k z}{\cosh k d} \cos \left(b_{0} z\right)\right\} d z \\
& \times \int_{0}^{d-h-b}\left\{\frac{\cosh k z}{\cosh k d} \cos \left(b_{0} z\right)\right\} d z \\
& \times \frac{1}{\int_{0}^{d-b-h}\left\{\cos \left(b_{0} z\right)\right\}^{2} d z} \\
& +\frac{J_{n}^{\prime}\left(k_{0} a_{j}\right)}{J_{n}\left(k_{0} a_{j}\right)} k_{0} \\
& \times\left\{\left[T_{j} J_{n}\left(k a_{j}\right) e^{i n(\pi / 2-\beta)}+C_{n}^{j} \frac{H_{n}\left(k a_{j}\right)}{H_{n}^{\prime}\left(k a_{j}\right)}\right.\right. \\
& +\sum_{\substack{l=1 \\
l \neq j}}^{N} \sum_{m=-\infty}^{\infty} C_{m}^{l} \frac{J_{n}\left(k a_{j}\right)}{H_{m}^{\prime}\left(k a_{l}\right)} \\
& \left.\left.\times H_{m-n}\left(k R_{l j}\right) e^{i(m-n) \alpha_{l j}}\right]\right\} \\
& \times \int_{d-h}^{d}\left\{\frac{\cosh k z}{\cosh k d} \frac{\cosh k_{0}(z-d+h)}{\cosh k_{0} h}\right\} d z
\end{aligned}
$$




$$
\begin{aligned}
& \times \int_{d-h}^{d}\left\{\frac{\cosh k z}{\cosh k d} \frac{\cosh k_{0}(z-d+h)}{\cosh k_{0} h}\right\} d z \\
& \times\left(1 \times\left(\left\{1+\frac{i J_{n}^{\prime}\left(k_{0} a_{j}\right)}{J_{n}\left(k_{0} a_{j}\right) G}\right\}\right.\right. \\
& \left.\left.\quad \times \int_{d-h}^{d}\left\{\frac{\cosh \left\{k_{0}(z-d+h)\right\}}{\cosh \left(k_{0} h\right)}\right\}^{2} d z\right)^{-1}\right) .
\end{aligned}
$$

In order to calculate the potential coefficients $C_{n}^{j}$ from the infinite system, $(24)$ is truncated to $(2 M+1) N$ equations with $(2 M+1) N$ unknown values for $j=1,2, \ldots, N$ and $n=-M, \ldots, M$. That is,

$$
\begin{aligned}
& C_{n}^{j}\left\{k \Gamma_{6}-\frac{|n|}{a_{j}(d-h-b)} \frac{H_{n}\left(k a_{j}\right)}{H_{n}^{\prime}\left(k a_{j}\right)} \Gamma_{1}\right. \\
& -\frac{H_{n}\left(k a_{j}\right)}{H_{n}^{\prime}\left(k a_{j}\right)} \frac{\Gamma_{2}}{\Gamma_{3}} \\
& \left.-\frac{J_{n}^{\prime}\left(k_{0} a_{j}\right)}{J_{n}\left(k_{0} a_{j}\right)} k_{0} \frac{H_{n}\left(k a_{j}\right)}{H_{n}^{\prime}\left(k a_{j}\right)} \frac{\Gamma_{4}}{\Theta_{1} \Gamma_{5}}\right\} \\
& +\sum_{\substack{l=1 \\
l \neq j}}^{N} \sum_{m=-M}^{M} C_{m}^{l} \frac{H_{m-n}\left(k R_{l j}\right)}{H_{m}^{\prime}\left(k a_{l}\right)} e^{i(m-n) \alpha_{l j}} \\
& \times\left\{J_{n}^{\prime}\left(k a_{j}\right) k \Gamma_{6}\right. \\
& -\frac{|n|}{a_{j}(d-h-b)} J_{n}\left(k a_{j}\right) \Gamma_{1} \\
& -J_{n}\left(k a_{j}\right) \frac{\Gamma_{2}}{\Gamma_{3}} \\
& \left.-\frac{J_{n}^{\prime}\left(k_{0} a_{j}\right)}{J_{n}\left(k_{0} a_{j}\right)} k_{0} J_{n}\left(k a_{j}\right) \frac{\Gamma_{4}}{\Theta_{1} \Gamma_{5}}\right\} \\
& =-T_{j} e^{i n(\pi / 2-\beta)} \\
& \times\left\{J_{n}^{\prime}\left(k a_{j}\right) k \Gamma_{6}-\frac{|n|}{a_{j}(d-h-b)} J_{n}\left(k a_{j}\right) \Gamma_{1}\right. \\
& \left.-J_{n}\left(k a_{j}\right) \frac{\Gamma_{2}}{\Gamma_{3}}-\frac{J_{n}^{\prime}\left(k_{0} a_{j}\right)}{J_{n}\left(k_{0} a_{j}\right)} k_{0} J_{n}\left(k a_{j}\right) \frac{\Gamma_{4}}{\Theta_{1} \Gamma_{5}}\right\} \text {, }
\end{aligned}
$$

where

$$
\begin{aligned}
& \Gamma_{1}=\int_{0}^{d-h-b} \frac{\cosh k z}{\cosh k d} d z \int_{0}^{d-h-b} \frac{\cosh k z}{\cosh k d} d z, \\
& \Gamma_{2}=\int_{0}^{d-h-b}\left\{\frac{\cosh k z}{\cosh k d} \cos \left(b_{0} z\right)\right\} d z
\end{aligned}
$$

$$
\begin{aligned}
& \times \int_{0}^{d-h-b}\left\{\frac{\cosh k z}{\cosh k d} \cos \left(b_{0} z\right)\right\} d z \times \sum_{q=1}^{\infty} \frac{I_{n}^{\prime}\left(b_{0} a_{j}\right) b_{0}}{I_{n}\left(b_{0} a_{j}\right)}, \\
\Gamma_{3}= & \int_{0}^{d-b-h}\left\{\cos \left(b_{0} x\right)\right\}^{2} d z, \\
\Gamma_{4}= & \int_{d-h}^{d}\left\{\frac{\cosh k_{0}(z-d+h)}{\cosh k_{0} h} \frac{\cosh k z}{\cosh k d}\right\} d z \\
& \times \int_{d-h}^{d}\left\{\frac{\cosh k_{0}(z-d+h)}{\cosh k_{0} h} \frac{\cosh k z}{\cosh k d}\right\} d z, \\
\Gamma_{5}= & \int_{d-h}^{d}\left\{\frac{\cosh k_{0}(z-d+h)}{\cosh k_{0} h}\right\}^{2} d z, \\
\Gamma_{6}= & \int_{0}^{d}\left\{\frac{\cosh k z}{\cosh k d}\right\}^{2} d z, \\
\Theta_{1}= & \left\{1+\frac{i J_{n}^{\prime}\left(k_{0} a_{j}\right)}{J_{n}\left(k_{0} a_{j}\right) G}\right\} .
\end{aligned}
$$

Using a standard matrix technique, the $(2 M+1) N$ equations for $C_{n}^{j}$ can be solved in (25), at which the unknown coefficients $A_{n}^{j}, B_{n, 0}^{j}$, and $B_{n, q}^{j}$ can be obtained from (19) and (21), after the calculated $C_{n}^{j}$. The procedure for deriving evanescent wave numbers $\left(k_{q}\right)$ is the same as that for deriving propagating wave numbers $(k)$. The velocity potential in each fluid region $\left(\phi_{1}^{j}, \phi_{2}^{j}, \phi_{3}^{j}\right)$ can thus be determined.

After solving the velocity potentials, the wave excitation forces on each cylinder can be obtained from integrating the pressure on the wetted surface. Surge $\left(F_{x}\right)$ and sway $\left(F_{y}\right)$ forces on both porous and rigid areas of each cylinder can be calculated as follows:

$$
\begin{array}{r}
F_{x}^{j}=-i \rho \omega \int_{d-h}^{d} \int_{0}^{2 \pi} \frac{-i g H}{2 \omega}\left\{\phi_{3}^{j}-\phi_{1}^{j}\right\} a_{j} \cos \theta d \theta d z, \\
F_{y}^{j}=-i \rho \omega \int_{d-h}^{d} \int_{0}^{2 \pi} \frac{-i g H}{2 \omega}\left\{\phi_{3}^{j}-\phi_{1}^{j}\right\} a_{j} \sin \theta d \theta d z, \\
\text { on } d-h \leq z \leq d, \\
F_{x}^{j}=-i \rho \omega \int_{d-h-b}^{d-h} \int_{0}^{2 \pi} \frac{-i g H}{2 \omega} \phi_{3}^{j} a_{j} \cos \theta d \theta d z, \\
F_{y}^{j}=-i \rho \omega \int_{d-h-b}^{d-h} \int_{0}^{2 \pi} \frac{-i g H}{2 \omega} \phi_{3}^{j} a_{j} \sin \theta d \theta d z, \\
\text { on } d-h-b \leq z \leq d-h,
\end{array}
$$

where (27) is for the porous area and (28) is for the impermeable portion of the cylinder, respectively. Heave force $\left(F_{z}\right)$ can also be calculated in the same manner, by integrating the pressure on the cylinder bottom:

$$
\begin{array}{r}
F_{z}^{j}=-i \rho \omega \int_{0}^{a} \int_{0}^{2 \pi} \frac{-i g H}{2 \omega}\left\{\phi_{2}^{j}-\phi_{1}^{j}\right\} r_{j} d \theta d r \\
\text { on } z=d-h-b .
\end{array}
$$




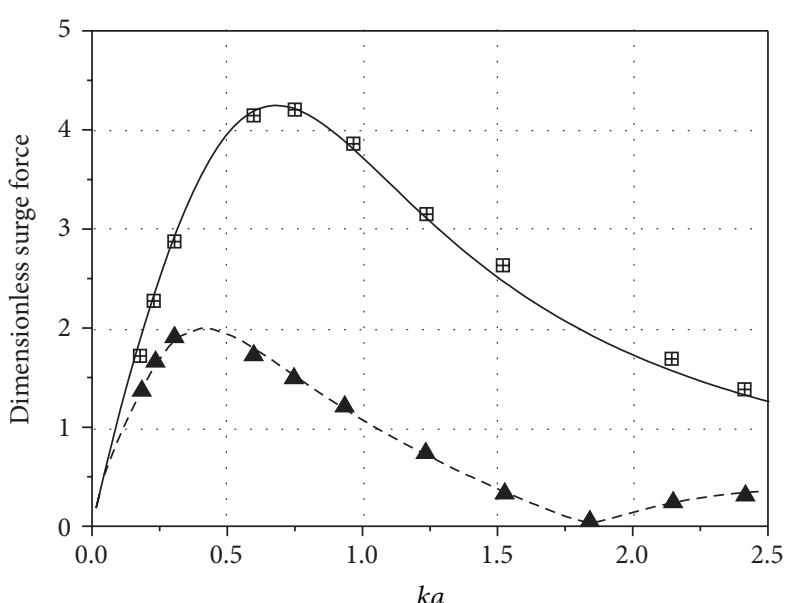

Zhao et al. (2011) 田 $\tau=0.0$ $\Delta \tau=0.14$
Present

$$
\text { - } G=0.0
$$$$
\text { - - } G=1.432
$$

(a)

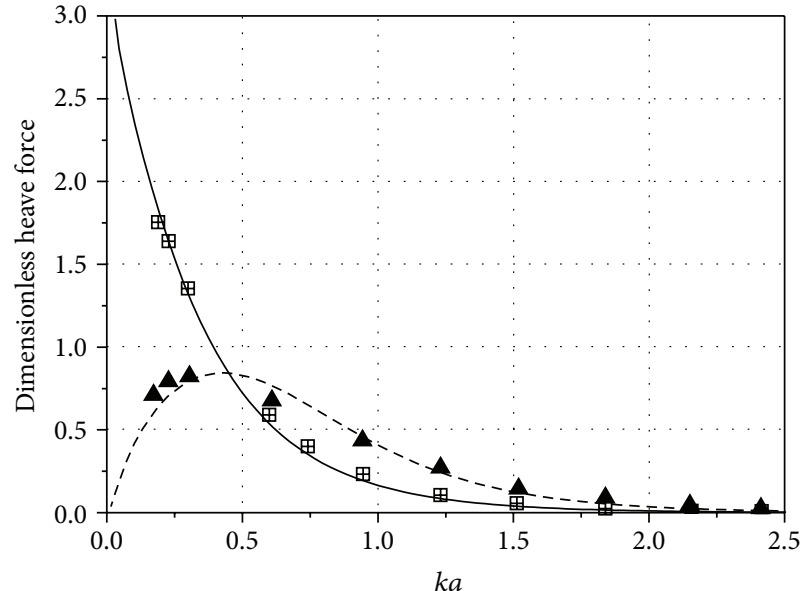

Zhao et al. (2011)

田 $\tau=0.0$

入 $\tau=0.14$

Present

- $G=0.0$

- - $G=1.432$

(b)

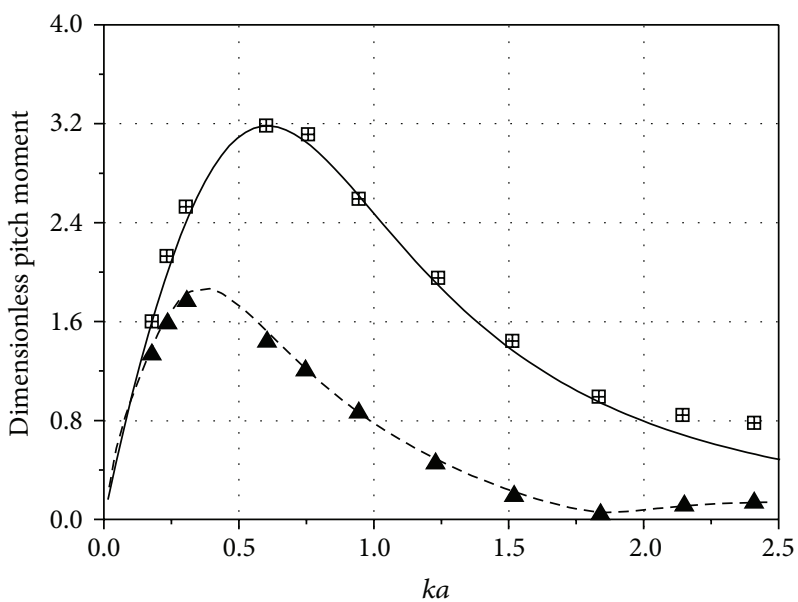

Zhao et al. (2011)

Present

田 $\tau=0.0$

$-G=0.0$

А $\tau=0.14$

$---G=1.432$

(c)

Figure 2: Comparison of numerical results with experimental results for $a=0.15 \mathrm{~m}, d=5 \mathrm{~m}, h=0.3 \mathrm{~m}, b=0.0 \mathrm{~m}, D=0.3 \mathrm{~m}$, and $\beta=0^{\circ}$ : (a) surge force, (b) heave force, and (c) pitch moment (see Park et al. [21] for surge and heave force comparison).

Pitch and roll moments are given by the relationship between the vertical force on the cylinder bottom and the horizontal forces on the cylinder wall:

$$
\begin{aligned}
M_{\text {pitch }}^{j}= & -\rho g \frac{H}{2} \int_{0}^{a} \int_{0}^{2 \pi}\left\{\phi_{2}^{j}-\phi_{1}^{j}\right\} r_{j}\left(X_{j}-r_{j} \cos \theta\right) d \theta d r \\
& -\rho g \frac{H}{2} \int_{d-h}^{d} \int_{0}^{2 \pi}\left\{\phi_{3}^{j}-\phi_{1}^{j}\right\} a_{j} \cos \theta d \theta(d-z) d z \\
& -\rho g \frac{H}{2} \int_{d-h-h}^{d-h} \int_{0}^{2 \pi}\left\{\phi_{3}^{j}\right\} a_{j} \cos \theta d \theta(d-z) d z, \\
M_{\text {roll }}^{j}= & -\rho g \frac{H}{2} \int_{0}^{a} \int_{0}^{2 \pi}\left\{\phi_{2}^{j}-\phi_{1}^{j}\right\} r_{j}\left(Y_{j}-r_{j} \sin \theta\right) d \theta d r
\end{aligned}
$$

$$
\begin{aligned}
& -\rho g \frac{H}{2} \int_{d-h}^{d} \int_{0}^{2 \pi}\left\{\phi_{3}^{j}-\phi_{1}^{j}\right\} a_{j} \sin \theta d \theta(d-z) d z \\
& -\rho g \frac{H}{2} \int_{d-h-b}^{d-h} \int_{0}^{2 \pi}\left\{\phi_{3}^{j}\right\} a_{j} \sin \theta d \theta(d-z) d z,
\end{aligned}
$$

where $X_{j}$ and $Y_{j}$ denote the global horizontal axes. Finally, the yaw moment on the $j$ th cylinder with respect to $X_{j}$ and $Y_{j}$ can be obtained as follows:

$$
M_{\text {yaw }}^{j}=-Y_{j} F_{x}^{j}+X_{j} F_{y}^{j}
$$




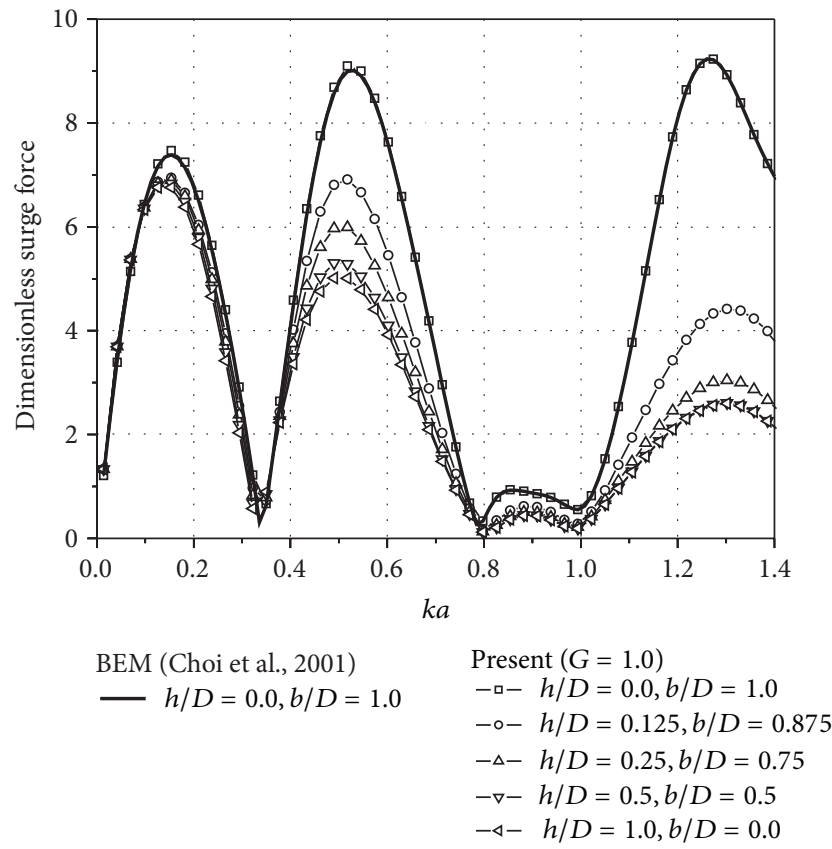

(a)

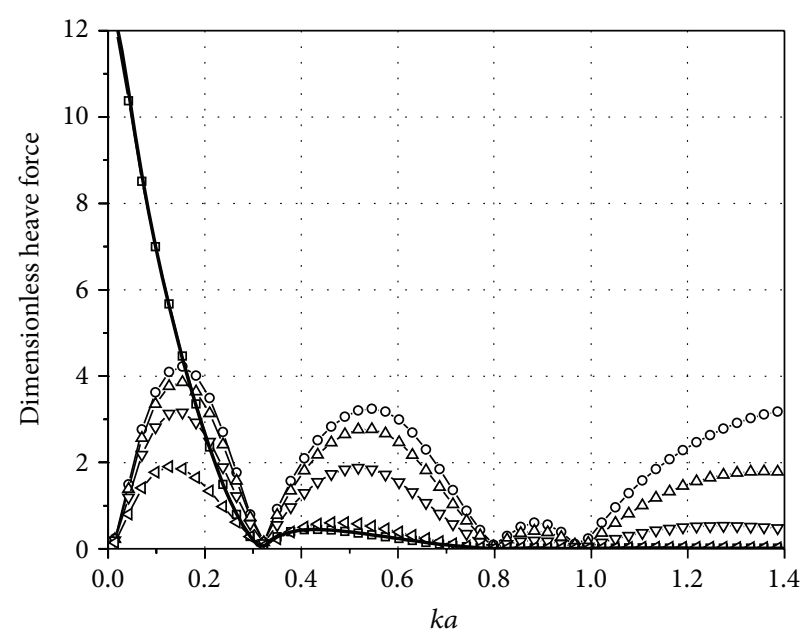

BEM (Choi et al., 2001)

Present $(G=1.0)$

- $h / D=0.0, b / D=1.0$

$-\square-h / D=0.0, b / D=1.0$

-o- $h / D=0.125, b / D=0.875$

$-\Delta-h / D=0.25, b / D=0.75$

$-\nabla-h / D=0.5, b / D=0.5$

$-\triangleleft-h / D=1.0, b / D=0.0$

(b)

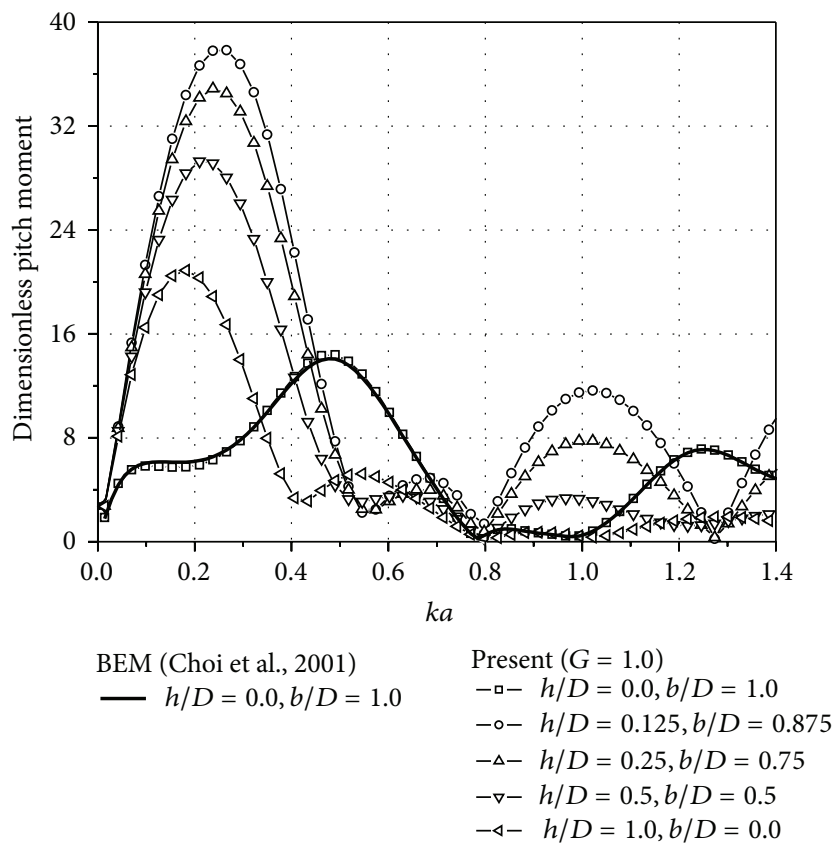

(c)

Figure 3: Comparison of total wave forces on four cylinders with $a=8.44 \mathrm{~m}, d=200 \mathrm{~m}, R=86.25 \mathrm{~m}, D=35 \mathrm{~m}, G=1.0$, and $\beta=22.5^{\circ}$ for various porosity depths (h): (a) surge force, (b) heave force, and (c) pitch moment.

\section{Results and Discussion}

In order to verify the wave forces on a single porous cylinder, the calculated results are compared with given experimental results of Zhao et al. [19] for the various incident wave frequencies shown in Figure 2. The computed body consists of a full-body porous cylinder with a very thin impermeable bottom plate, a radius $(a)$ of $0.15 \mathrm{~m}$, and porous-wall $(h)$ of $0.3 \mathrm{~m}(D=0.3 \mathrm{~m})$. The water depth $(d)$ in the computational domain is $5 \mathrm{~m}$ and the incident wave angle $(\beta)$ is $0^{\circ}$. The calculated surge and heave forces are normalized by $\rho g(H / 2) a^{2}$ and the pitch moment is normalized by $\rho g(H / 2) a^{3}$. Note that all subsequent calculation results in this study are normalized by the aforementioned forms. For comparison in Figure 2, based on the relationship between the porosity parameter $(G)$ and the opening ratio $(\tau)$ in Zhao et al. [19], $G=(17.8 / \varepsilon+$ $143.2) \tau^{2} / 2 \pi(1+1.06 \tau)$, and the parameter $(G)$ of 1.432 is 

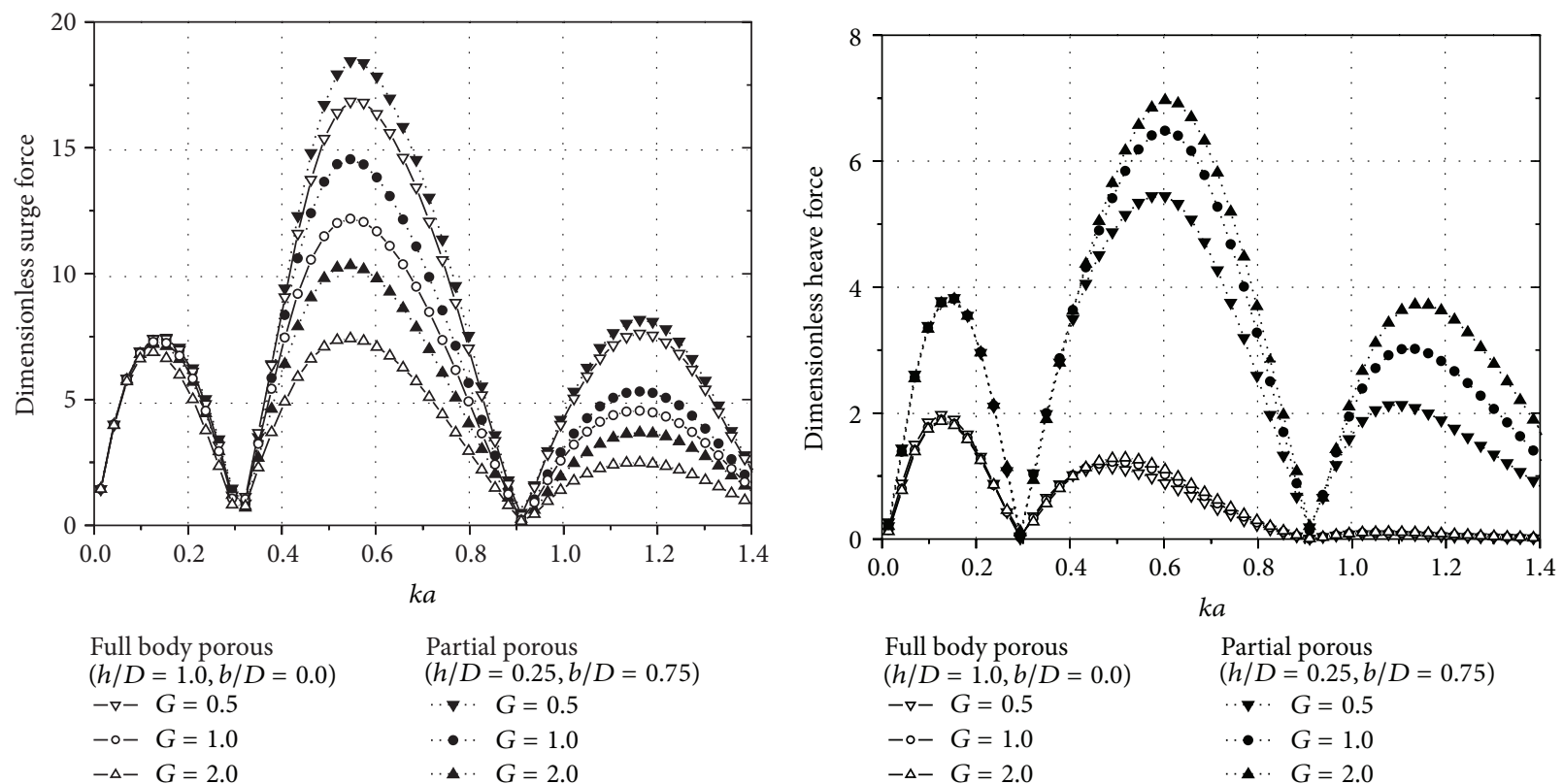

(a)

(b)

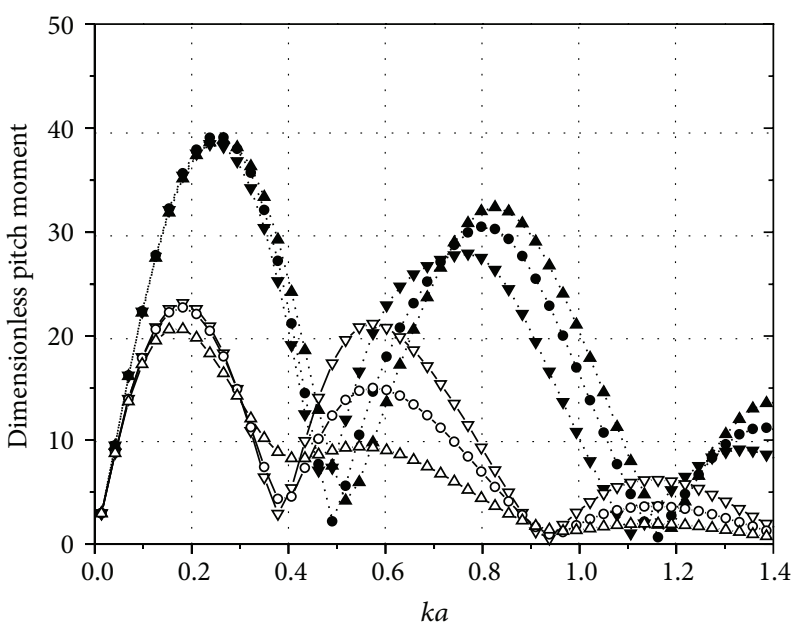

$$
\begin{array}{ll}
\begin{array}{l}
\text { Full body porous } \\
(h / D=1.0, b / D=0.0)
\end{array} & \begin{array}{l}
\text { Partial porous } \\
(h / D=0.25, b / D=0.75)
\end{array} \\
-\nabla-G=0.5 & \cdots \vee \cdots G=0.5 \\
-\circ-G=1.0 & \cdots \bullet \cdot G=1.0 \\
-\Delta-G=2.0 & \cdots \backsim G G=2.0
\end{array}
$$

(c)

Figure 4: Wave forces on four cylinders with $\beta=0.0^{\circ}$ for various porosity parameters $(G)$ : (a) surge force, (b) heave force, and (c) pitch moment. Body geometry is the same as Figure 3.

used for the present comparison, where the opening ratio $(\tau)$ is 0.14 (equivalent to $G=1.432$ ) and the wave steepness $(\varepsilon)$ is 0.04633 for the experimental data. Both the numerical and experimental results appear to agree. The wave loads on an impermeable body $(G=0)$ in the respective directions are generally greater than those on a porous-surfaced body, since the incident wave energy is absorbed by the pores on the cylinder wall. The surge and pitch wave loads on the porous cylinder are close to zero when $\mathrm{ka}$ is approximately 1.84 , at which the first nontrivial zero of the derivative of the
Bessel function $J_{n}^{\prime}(k a)$ exists. It is also observed that the peak frequencies of the surge and pitch modes for the porous body are lower than those for the impermeable body, suggesting that the structural properties of the system have been changed due to porosity on the cylinder wall. The decrease of vertical force on the porous body (second graph in Figure 2) is remarkable when compared to that of the rigid cylinder, going to zero when $k a$ is less than 0.5 and slightly increasing when $k a$ is greater than 0.5 . From this comparison, it is apparent that the vertical force in the long wave region is significantly 

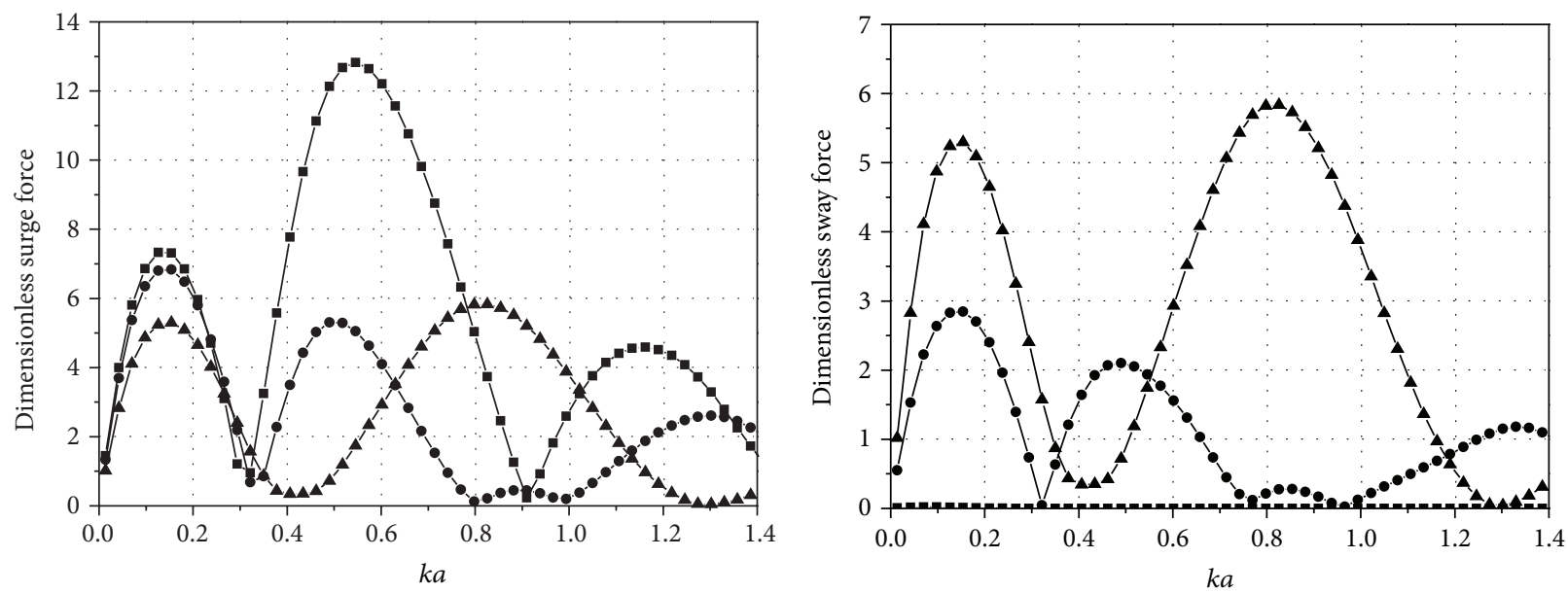

Wave angle $(\beta)$
$--\beta=0.0$
$-\bullet-\beta=22.5$
$-\mathbf{-}-\beta=45.0$

Wave angle $(\beta)$

$-\boldsymbol{-}-\beta=0.0$

$-\bullet-\beta=22.5$

$-\mathbf{\Delta}-\beta=45.0$

(a)

(b)

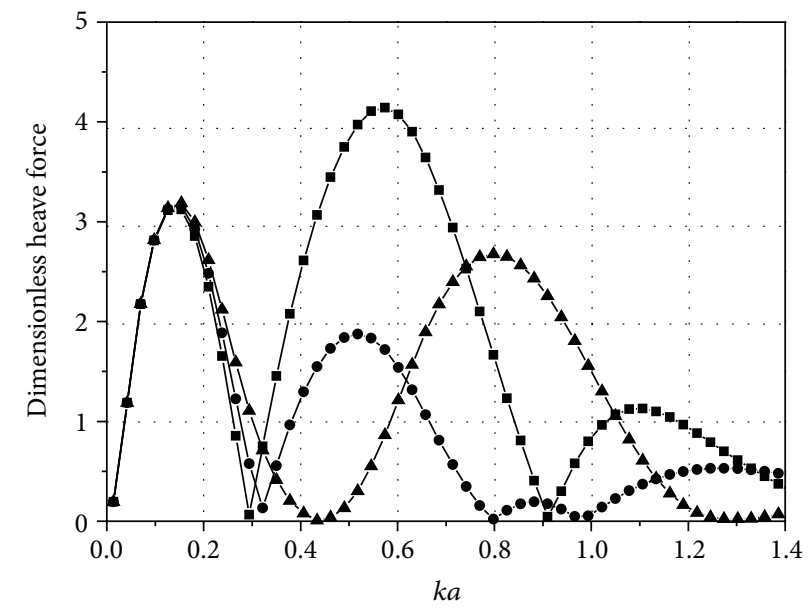

$$
\begin{aligned}
& \text { Wave angle }(\beta) \\
& --\beta=0.0 \\
& -\bullet-\beta=22.5 \\
& -\mathbf{-}-\beta=45.0
\end{aligned}
$$

(c)

FIGURE 5: Wave forces on four partial-porous cylinders with $h / D=0.5, b / D=0.5$, and $G=1.0$ for different incident wave angles $(\beta)$ : (a) surge force, (b) sway force, and (c) heave force; other conditions are the same as Figure 3.

influenced by the porosity of the side wall. This data suggests that vertical wave loads from long waves could be effectively controlled by installing porous materials on the cylinder walls. This comparison was also found in Park et al. [21], in which the numerical analysis of the multi-porous cylinders with wave excitation and seismic forces was performed.

Figure 3 shows the comparison of wave forces on four cylinders for various porous wall-lengths $(h)$. The calculation conditions are $a=8.44 \mathrm{~m}, d=200 \mathrm{~m}, D=35 \mathrm{~m}, G=1.0$, and $\beta=22.5^{\circ}$. The cylinders are numbered clockwise from one to four and situated at $(-43.125,43.125) \mathrm{m},(43.125,43.125) \mathrm{m}$, $(43.125,-43.125) \mathrm{m}$, and $(-43.125,-43.125) \mathrm{m}$, respectively.
The distance between the cylinder centers $(R)$ is $86.25 \mathrm{~m}$, which is approximately five times the cylinder diameter $(R /(2 a) \approx 5)$. In comparison, the ratio of $h / D=0.0$ and $b / D=1.0$ represents the full-body impermeable cylinder, while the ratio of $h / D=1.0$ and $b / D=0.0$ represents the full-body porous cylinder. The calculated wave forces of the full-rigid body agree well with the results from HOBEM [23]. Since the strength of the wave-body interaction is closely related to the ratio of the wavelength to the distance between bodies, the modulation of force-magnitude is observed over a given range of wave frequencies. When the porous-length ratio $(h / D)$ is only $12.5 \%$ of the entire draft $(h / D=0.125)$, 


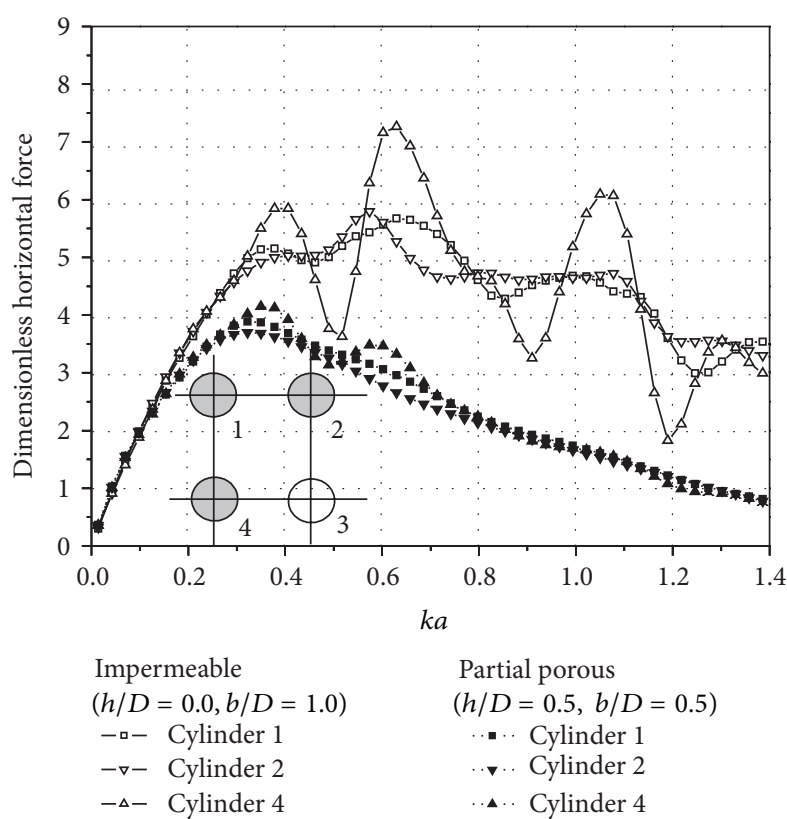

(a)

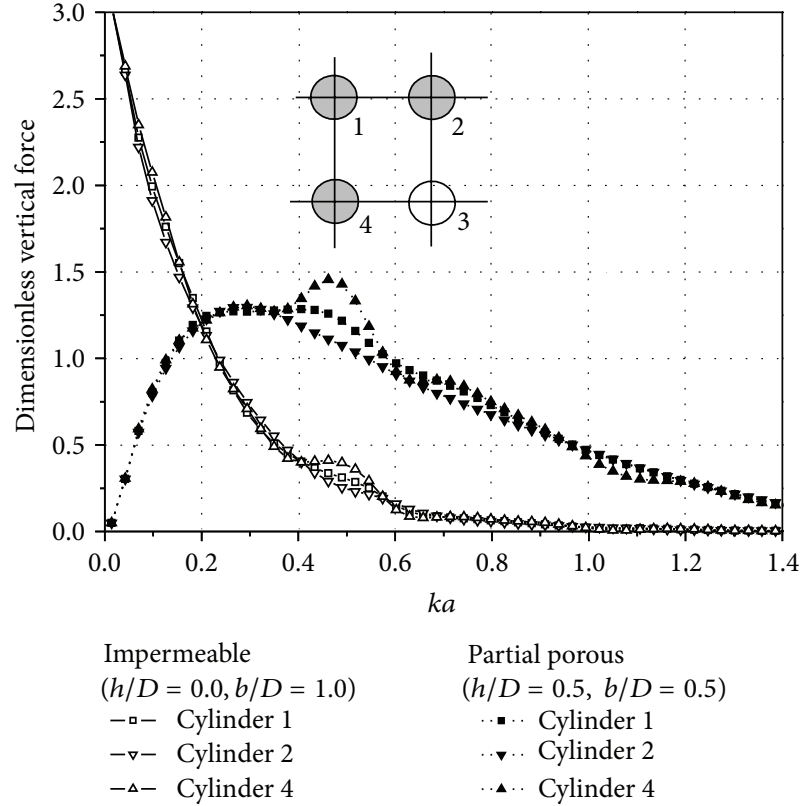

(b)

FIGURE 6: Comparison of wave force on each cylinder with $\beta=45.0^{\circ}$ : (a) horizontal force $\left(\sqrt{F_{x}^{2}+F_{y}^{2}}\right)$ and (b) vertical (heave) force $\left(F_{z}\right)$; other conditions are the same as Figure 3.

the forces at the second and fourth peaks (at $k a=0.5$ and $k a=1.3$ ) decrease by approximately $23 \%$ and $53 \%$, respectively, as compared to the rigid body. The reduction rate of the surge force then gradually decreases when the porouslength ratio $(h / D)$ is greater than 0.125 . For instance, the final surge force of the full-body porous cylinder $(h / D=1.0)$ at the second peak $(k a=0.5)$ is approximately $40 \%$ of the force for the full-rigid cylinder $(b / D=1.0)$. This indicates that the influence of porosity rapidly decreases as the effective water depth increases. Conversely, the heave force pattern of partial-porous bodies is observed to be distinct from that of fully rigid bodies. In the long wave region, the vertical forces on the partial-porous body greatly decrease and converge to zero as the wave stiffness $(k a)$ goes to zero. As the ratio of porous-length $(h / D)$ decreases, the magnitude of vertical force increases. This begins to more closely resemble that of the rigid body, where the value of vertical force was confirmed after the convergence test. The heave forces of the porous body are also modulated over a given frequency range, while those of the rigid body decrease monotonically. Owing to the influence of the surge and heave forces, the pattern of pitch moments for the partial-porous body is similar to that of the other forces. Consequently, a circular cylinder with a small porous-length wall near the free surface could effectively reduce wave loads and resultant hydrodynamic interaction.

In order to examine the effect of porosity strength, the respective wave forces on four full-porous and partial-porous cylinders for various porosity parameters $(G)$ are presented in Figure 4 . The case of $h / D=0.25$ and $b / D=0.75$ was selected for the partial-porous body (i.e., the porous wall-length is $25 \%$ of the entire draft). It is obvious that the surge forces on the full-body porous cylinders are generally smaller than those on the partial-porous cylinders. Moreover, no great deviation between the partial- and full-porous cases can be seen near the long wave region (the first mode frequency, $k a \leq 0.3)$. In the second and third peak area, the magnitude of forces decreases with each increment of porosity strength.

Heave forces on partial-porous bodies are much greater than those on full-body porous cylinders, regardless of porosity strength. For the vertical wave loads observed in Figures 3 and 4 , the influence of porous wall-length is more critical than that of porosity strength $(G)$. As porosity strength increases, heave force gradually increases, which is contrary to expectations from the surge force results. This observation may be explained by the fact that the effect of the wave potential at the upper rigid top of the cylinder in region $1(z=d-h)$ becomes significant, so it can support the vertical force as the porosity increases. In other words, the interaction between the wave and the porous body may intensify the hydrodynamic force on the top of the rigid cylinder (region 1) with a large porosity. Due to the influence of translational and rotational loads, the pattern of pitch moments can also be a combination of both surge and heave forces.

Figure 5 shows the comparison of wave forces on four partial-porous cylinders $(h / D=0.5, b / D=0.5)$ with various incident wave angles $(\beta)$. This figure shows that the magnitude of hydrodynamic force as a function of wave frequency is greatly influenced by the angle of incident waves. In addition, the translational wave loads (surge and sway forces) are strongly dependent on incident wave angles owing to individual body location. It is obvious that the maximum surge force occurs at the wave angle of $0^{\circ}$, normal to the cylinder array, at which the minimum sway force is simultaneously observed due to the symmetry of body 


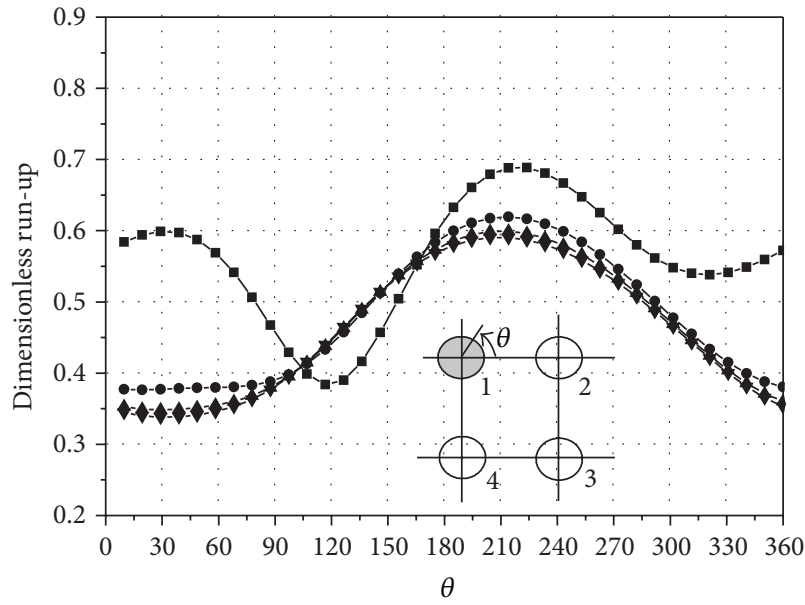

Cylinder 1

$$
\begin{aligned}
& \text { - } h / D=0.0, b / D=1.0 \quad-\Delta-h / D=0.5, b / D=0.5 \\
& -\bullet-h / D=0.25, b / D=0.75 \quad-v-h / D=1.0, b / D=0.0
\end{aligned}
$$

(a)

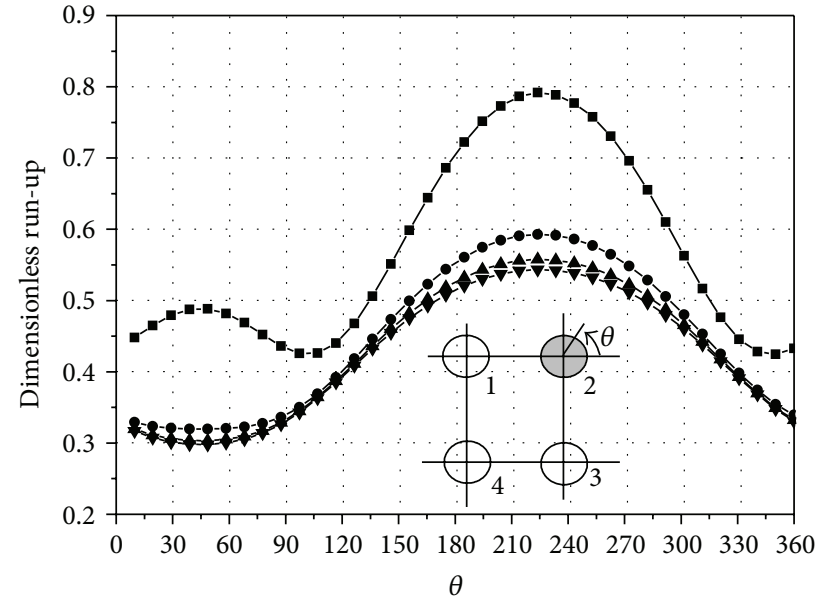

Cylinder 2

$\begin{array}{ll}-h / D=0.0, b / D=1.0 & -\Delta-h / D=0.5, b / D=0.5 \\ -\bullet-h / D=0.25, b / D=0.75 & -\bullet-h / D=1.0, b / D=0.0\end{array}$

(b)

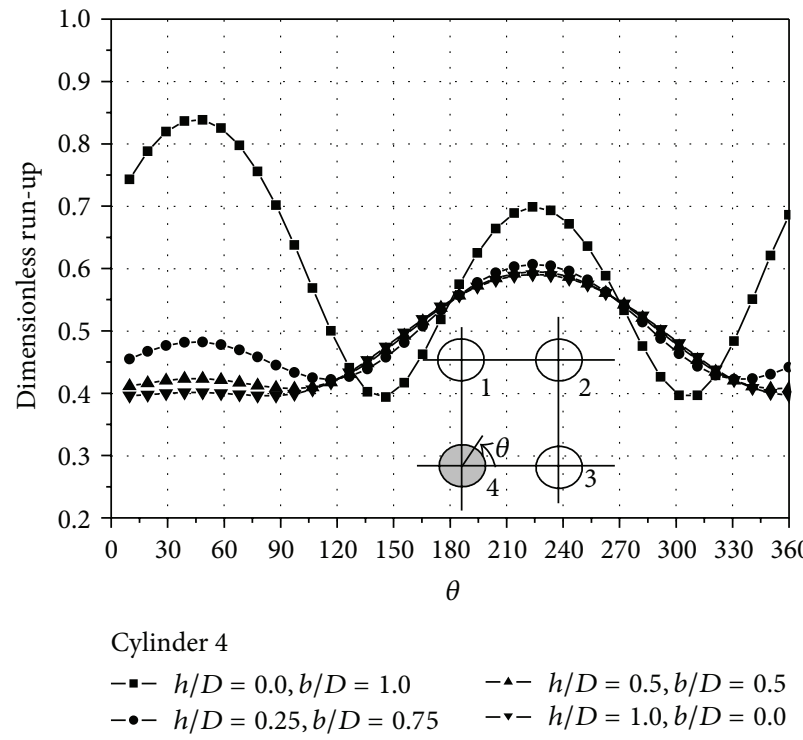

(c)

Figure 7: Comparison of wave run-up on each cylinder for various porosity depths with $\beta=45.0^{\circ}, G=1.0$, and $k a=0.6$ : (a) cylinder 1 , (b) cylinder 2, and (c) cylinder 4; the value is normalized by incident wave height $(H)$. Other conditions are the same as Figure 3.

positions. The variation of heave forces shows a pattern similar to that of the surge forces as $k a$ increases, showing that the hydrodynamic interaction with waves of the same direction may also be dominant for vertical wave loads.

In order to evaluate the wave forces on each cylinder at different locations with an incident wave angle of $45^{\circ}$, the horizontal and vertical forces on full-rigid and partialporous cylinders are compared in Figure 6. The horizontal force $\left(\sqrt{F_{x}^{2}+F_{y}^{2}}\right)$ is composed of the surge $\left(F_{x}\right)$ and sway force $\left(F_{y}\right)$. In the case of $k a>0.2$, the horizontal wave forces on full-rigid cylinders are greater than those on partial-porous bodies at all locations, while the vertical forces on full-rigid bodies are smaller than those on partial-porous cylinders as shown in Figure 3(b). For cylinder 4, located in front of the wave ray, the horizontal forces of the full-rigid body show modulated peaks at certain wave frequencies $(k a \approx$ $0.4,0.63$, and 1.05), and the maximum force is observed at $k a=0.63$, where the corresponding wavelength is similar to the body distance $(R)$. It is noted that the strength of the wave-body interaction varies in relation to the wavelength and the body distance $(R)$. The force on a partial-porous body $(h / D=0.5, b / D=0.5)$, however, has little variation and shows a monotonic change, meaning that the hydrodynamic interaction with the partial-porous body is much less than that with the full-rigid body. From this comparison, it is inferred that the waves scattered by the cylinders are rapidly dampened as they pass through the porous surface of the 

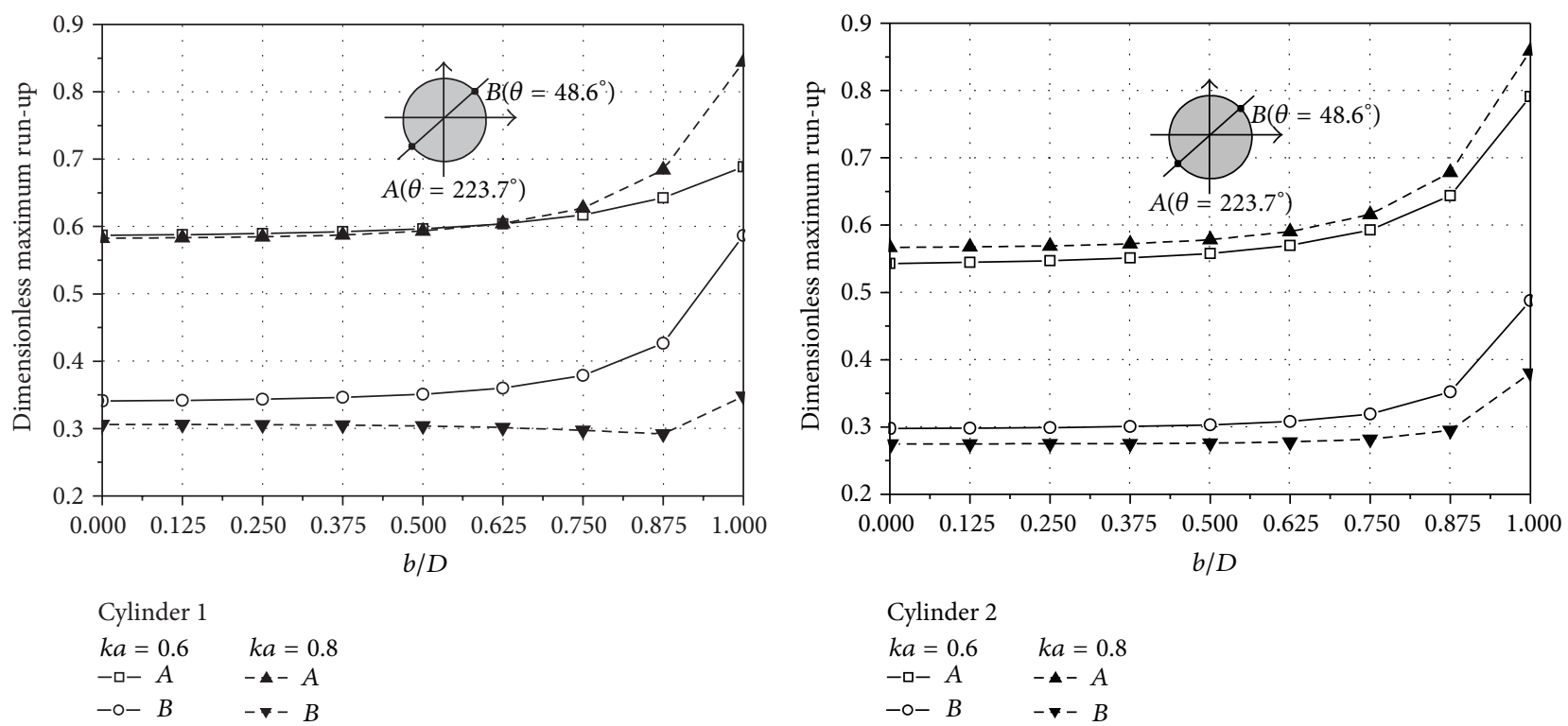

(a)

(b)

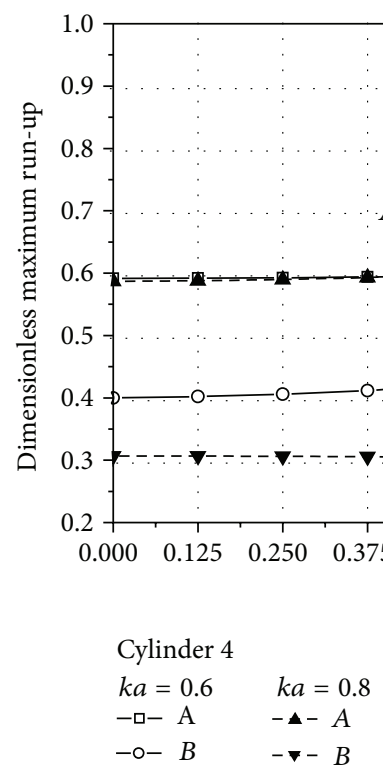

(c)

Figure 8: Comparison of maximum wave run-up at two locations with $\beta=45.0^{\circ}, G=1.0$, and $k a=0.6$ for various rigid-wall depths $(b / D)$ : (a) cylinder 1, (b) cylinder 2, and (c) cylinder 4; other conditions are the same as Figure 3.

cylinder. Therefore, the partial-porous body can be effectively used for multibodied structures requiring minimal wave interference between bodies.

Figure 7 shows a comparison of wave run-up on each cylinder for the full-rigid and partial-porous bodies. The wave run-up has been normalized by the incident wave height $(H)$, and the $x$-axis denotes the angle $(\theta)$ measured counterclockwise from the positive $x$-axis. Since the magnitude of hydrodynamic interaction decreases in the porous bodies, as was discussed in Figure 6, the maximum run-up on partialporous bodies is generally lower than that on the full-rigid bodies. For cylinders 1 and 2 (Figures $7(\mathrm{a})$ and $7(\mathrm{~b})$ ), the maximum run-up values for both surfaced-body conditions occur at a similar location, around the incident wave angle of $223.7^{\circ}$. On the other hand, the maximum run-up for the rigidsurfaced cylinder 4 occurs at the rear of the body $\left(\theta=48.6^{\circ}\right)$, against wave propagation direction, and the second peak is observed at the front of the body $\left(223.7^{\circ}\right)$. This phenomenon implies that the strong hydrodynamic interaction between four cylinders can magnify the wave run-up at the rear of the foremost cylinder. However, for partial-porous cylinders, the pattern of wave run-up for respective bodies is not sensitive to body location because of the reduction of wave-body interactions. 


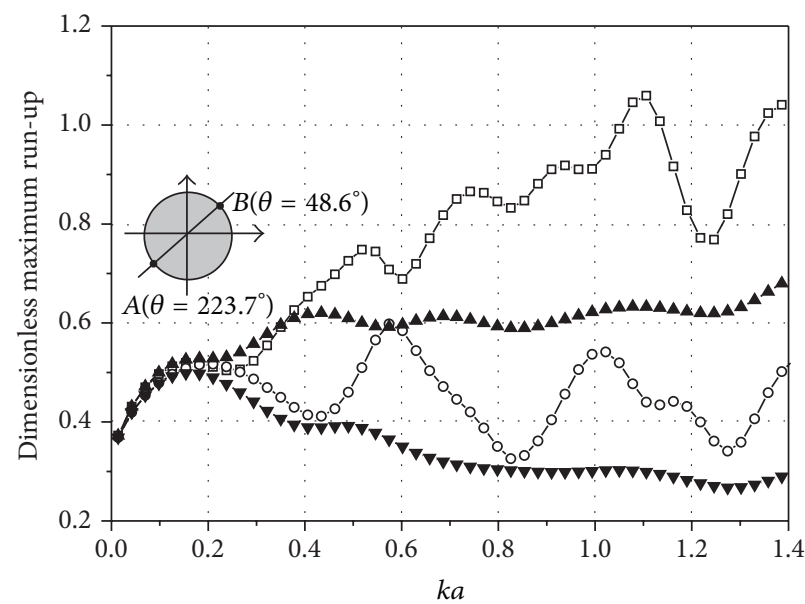

Cylinder 1

$$
\begin{array}{ll}
(h / D=0.0, b / D=1.0) & (h / D=0.5, b / D=0.5) \\
-\square-A & -\triangle-A \\
-\circ-B & --B
\end{array}
$$

(a)

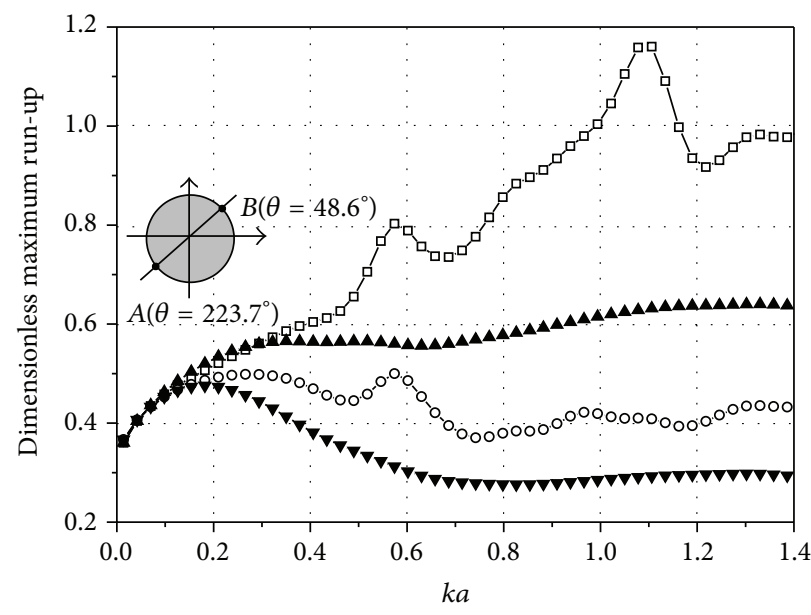

Cylinder 2

$$
\begin{array}{ll}
(h / D=0.0, b / D=1.0) & (h / D=0.5, b / D=0.5) \\
-\square-A & -A \\
-0-B & -
\end{array}
$$

(b)

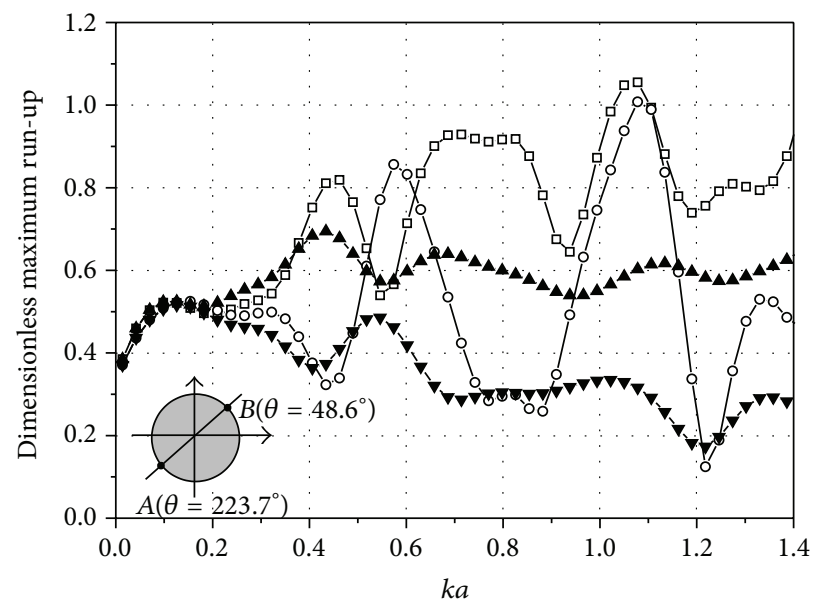

Cylinder 4

$$
\begin{array}{ll}
(h / D=0.0, b / D=1.0) & (h / D=0.5, b / D=0.5) \\
-\square-A & -\triangle-A \\
-\circ-B & -\checkmark-B
\end{array}
$$

(c)

FIGURE 9: Comparison of maximum wave run-up on four cylinders with $\beta=45.0^{\circ}$ and $G=1.0$ : (a) cylinder 1, (b) cylinder 2, and (c) cylinder 4; other conditions are the same as Figure 3.

In order to evaluate the effect of the porous wall-depth $(h)$ on the wave run-up, the results for various rigid walllength ratios $(b / D)$ are compared in Figure 8 . The measurement points $\mathrm{A}$ and $\mathrm{B}$ are selected at $\theta=223.7^{\circ}$ and $\theta=$ $48.6^{\circ}$, respectively, from the observed peak values shown in Figure 7. The $x$-axis value $(b / D)$ from 0.0 to 1.0 denotes a fulldepth porous cylinder to a full-depth impermeable cylinder. It is observed that the height of wave run-up for a given wave condition does not significantly increase until the ratio of rigid wall-length $(b / D)$ is 0.75 . For instance, the run-up height increases less than $15 \%$ from the case of the full-porous body $(b / D=0.0)$ until the porous wall-length becomes $25 \%$ of the total submerged cylinder wall $(h / D=0.25$ or $b / D=$ $0.75)$. In other words, the role of porosity in reducing runup height may be retained when the porous wall is installed at only a quarter of the total body draft. The increase in rate of run-up height for cylinder 4 at point B (the rear side of the cylinder) is found to be greatest as the rigid-wall ratio $(b / D)$ increases, at which the wave-body interaction becomes significant on the foremost cylinder, which was shown in Figure 7(c).

In Figure 9, the maximum run-up heights for respective cylinders are compared with various wave frequencies. Cylinder 4 is situated in front of cylinder 2 at an incident 


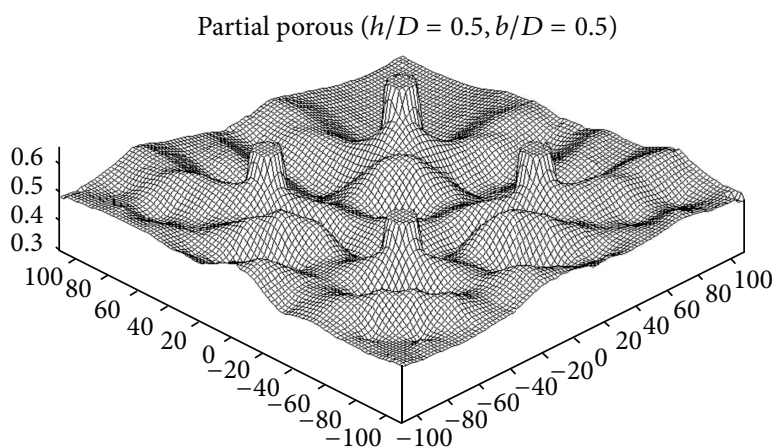

(a)

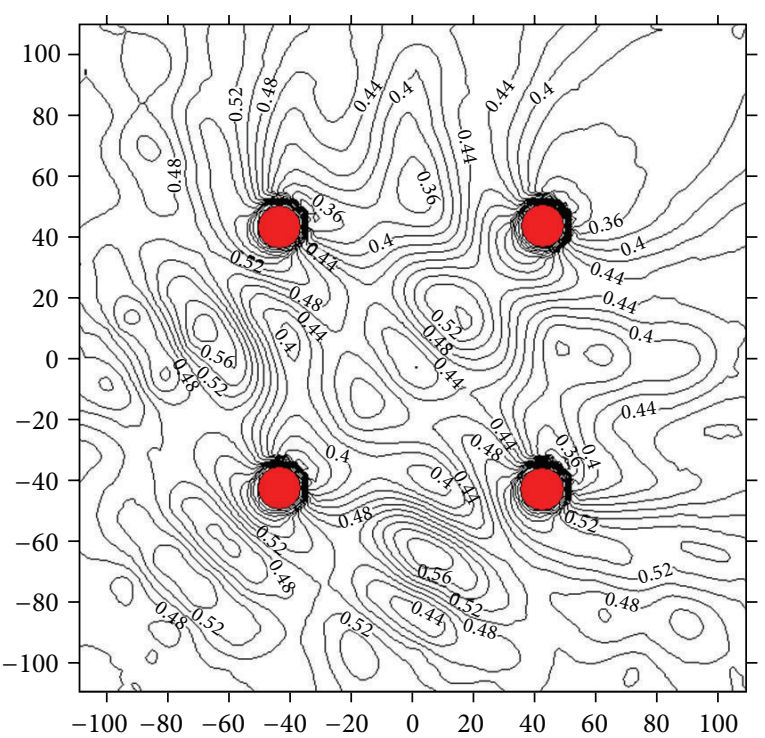

(b)

Figure 10: Snapshot of free surface elevations for four partial-porous cylinders with $h / D=0.5, b / D=0.5, k a=0.8$, and $\beta=45.0^{\circ}$; other conditions are the same as Figure 3.

wave angle of $45^{\circ}$. The run-up height for the full-rigid body $(h / D=0.0, b / D=1.0)$ is generally greater than that for the partial-porous body when $k a>0.2$. Depending on the relative distance between body gap and wavelength, the runup heights on the full-rigid cylinders show modulated values. Because of their relatively small wave-body interaction, a lesser fluctuation or even a monotonous change of run-up (less than 0.7 ) is observed in the partial-porous cylinders. The maximum wave run-up occurs at $k a \approx 1.1$ for all cylinders, where the corresponding wavelength is similar to the gap distance between cylinders 1 and 4 in the direction of wave propagation.

Figure 10 presents a snapshot of the free-surface elevation around the cylinders $(h / D=0.5, b / D=0.5)$ for $k a=0.8$ and $\beta=45^{\circ}$. Higher wave elevations are observed at the center of the four cylinders as well as at the front and rear of the cylinders against the wave direction. This snapshot highlights the locations of strong hydrodynamic interaction with the multibodied structures.

Figure 11 shows the total forces on four partial-porous cylinders for various gap distances $(R)$ between cylinders. The magnitude of the calculated force is modulated, and several peaks are observed according to wave frequencies. The location of the modulated peaks also varies depending on the gap distance. In the case of $R / 2 a=4$, which is the total structure width $\left(R^{\prime}=10 a\right)$, the peaks of surge and heave forces are observed at the wavelength of $R^{\prime}(k a \approx 0.65)$ and $4 R^{\prime}(k a \approx 0.17)$. The minimums are at $0.5 R^{\prime}(k a \approx 1.17)$ and $1.5 R^{\prime}(k a \approx 0.4)$, respectively. This phenomenon is also seen in other cases such as $R / 2 a=2$ and $R / 2 a=3$. In particular, for the case of $R / 2 a=1$, where the gap distance is zero and all cylinders are stuck together, no force modulation can be found, but the highest force magnitude is observed.
The average wave forces for four-cylinder and ninecylinder structures are compared in Figure 12. The average force is calculated from the total force of all cylinders divided by the number of bodies $(N)$. The average force on four cylinders is found to be similar to that on nine cylinders for the respective directions. The maximum surge forces on both four and nine cylinders are found at $k a \approx 0.6$, which are similar to the cases seen in Figure 4(a), and the maximum heave forces on both rigid cases are found in lower frequencies, as in Figure 6(b). As seen in Figure 11, the force magnitudes tend to vary with respect to wave frequency (relative wavelength to body gap distance); thus, the force can be modulated and have more peaks as the number of bodies increases.

\section{Conclusions}

Under the assumption of potential flow and linear wave theory, a 3D numerical method for an array of surfacepiercing partial-porous stationary cylinders was developed using the eigenfunction expansion method and Darcy's law, with a complete form of the mathematical formulations. This model was used to scrutinize the hydrodynamic properties of $N$ circular partial-porous cylinders, combined with three computational fluid domains. This method can be used to evaluate the hydrodynamic properties of any type of circular cylinder, including full-rigid, full-porous, and partial-porous bodies. This method was verified and found to agree with experimental data for a single porous cylinder and with the results of high-order boundary element method for a full-rigid cylinder. The horizontal force on a partial-porous cylinder was generally found to be small in comparison to that on a rigid body. In the long wave region, a great reduction 

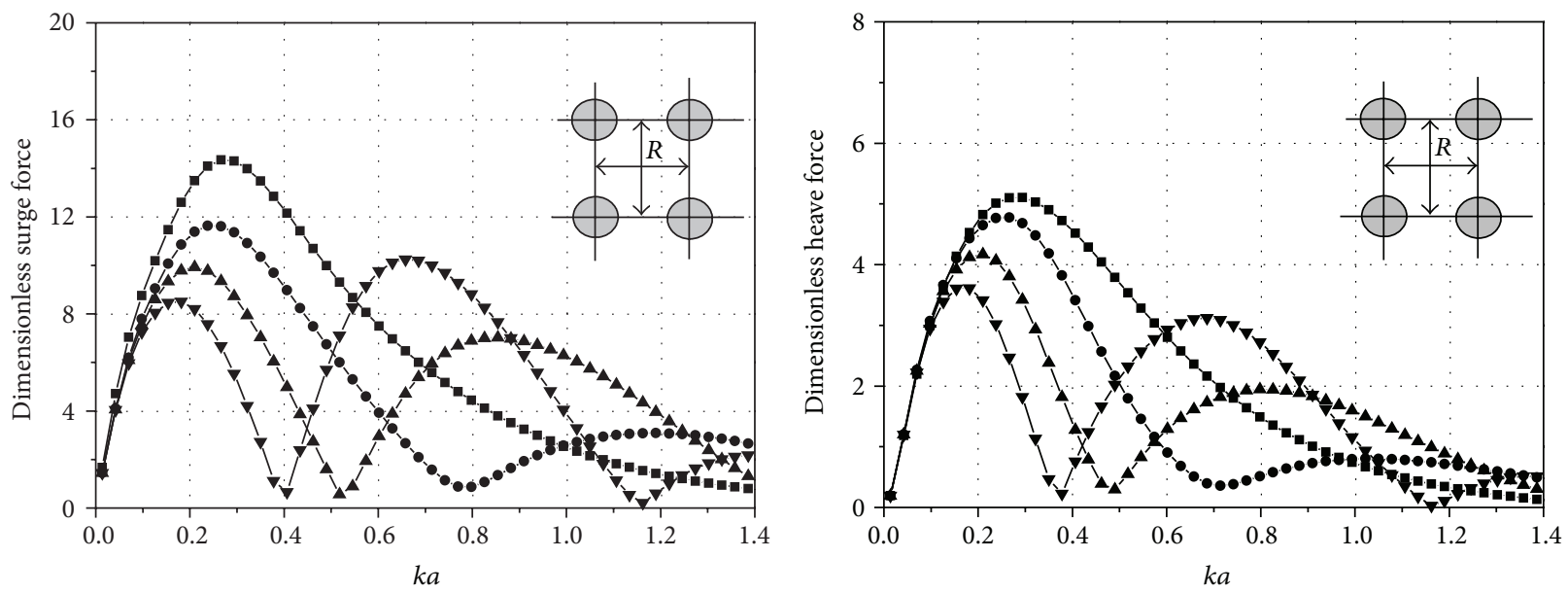

$$
\begin{aligned}
& \text { Partial porous } \\
& \begin{array}{ll}
(h / D=0.5, b / D=0.5) & \\
--R /(2 a)=1 & -\mathbf{-}-R /(2 a)=3 \\
-\bullet-R /(2 a)=2 & -\boldsymbol{\nabla}-R /(2 a)=4
\end{array}
\end{aligned}
$$

(a)
Partial porous

$(h / D=0.5, b / D=0.5)$

$\begin{array}{ll}\text { - } R /(2 a)=1 & -\mathbf{-}-R /(2 a)=3 \\ -\bullet-R /(2 a)=2 & -\boldsymbol{\nabla}-R /(2 a)=4\end{array}$

(b)

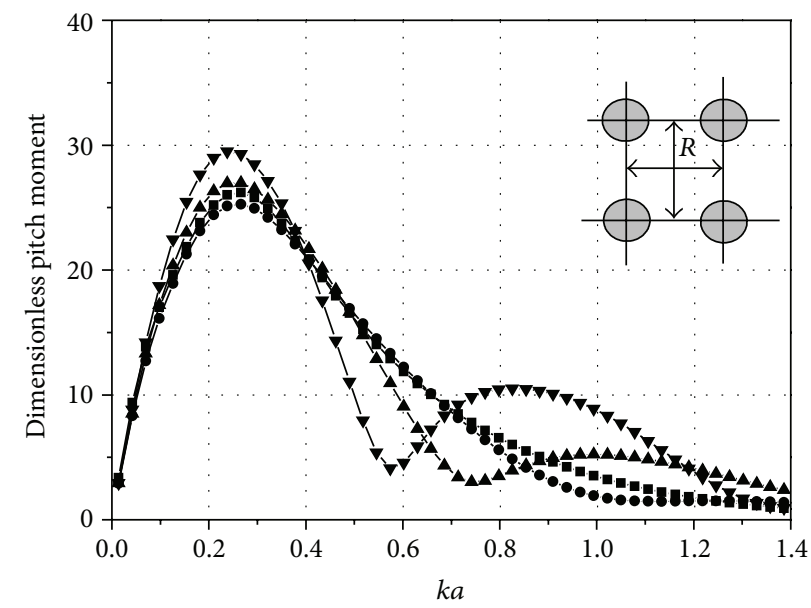

Partial porous

$(h / D=0.5, b / D=0.5)$

$-\mathbf{-} R /(2 a)=1 \quad-\mathbf{-}-R /(2 a)=3$

$-\bullet-R /(2 a)=2 \quad-\nabla-R /(2 a)=4$

(c)

Figure 11: Comparison of wave forces on four partial-porous cylinders for various cylinder gap distances $(R), \beta=0.0^{\circ}$ : (a) surge force, (b) heave force, and (c) pitch moment; other conditions are the same as Figure 3.

of the vertical force on the partial-porous body was observed compared to that on the rigid body. This phenomenon was confirmed by a convergence test. These data indicate that installing porous materials on the cylinder wall near the free surface could be an effective means of controlling the horizontal and vertical wave loads. The scattered waves generated by the four partial-porous cylinders were rapidly diminished as the waves passed through the porous surface of the body. This effect could be applied to situations where minimal wave interference between bodies is required.
The greatest wave run-up for the four rigid bodies was observed at the rear of the foremost cylinder against wave propagation direction, implying that the hydrodynamic interaction between four cylinders can magnify the wave run-up. The run-up for the partial-porous cylinder was less influenced by the cylinder location, however, because of the reduction in wave-body interaction. The role of porosity in diminishing the run-up height may be retained when the porous-surfaced wall is installed to only a quarter of the total body draft. Finally, this method for an array of partial-porous 

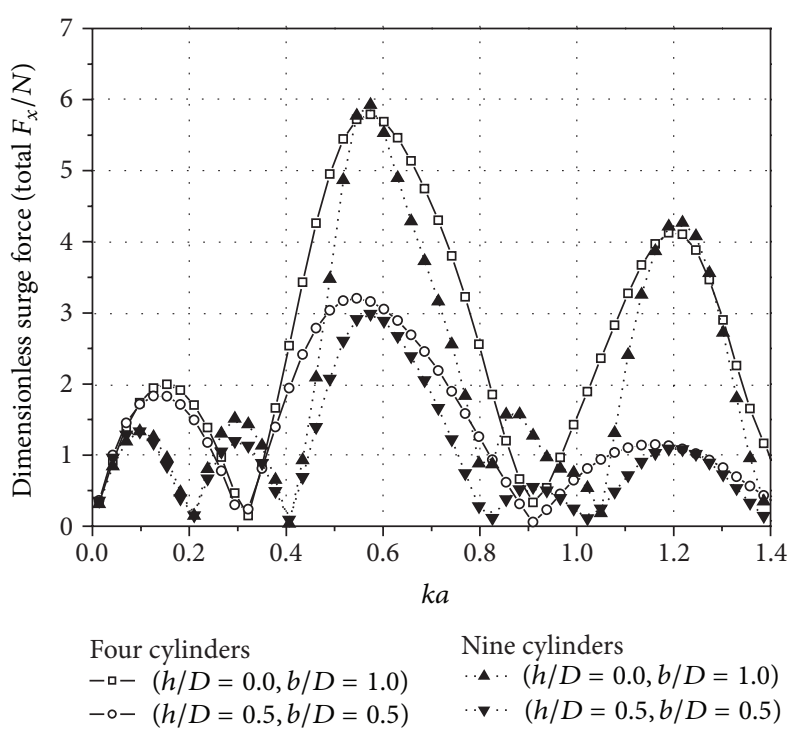

(a)

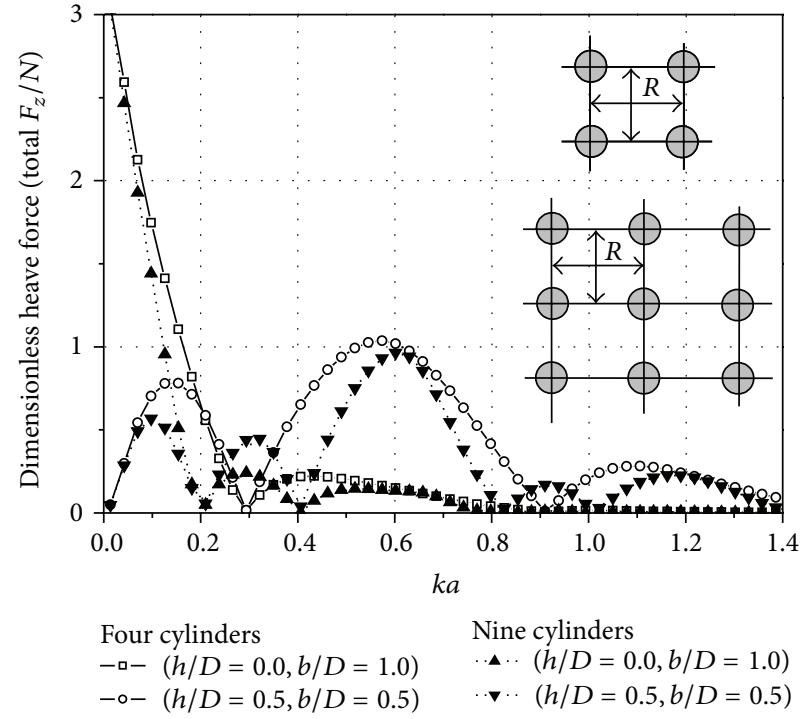

(b)

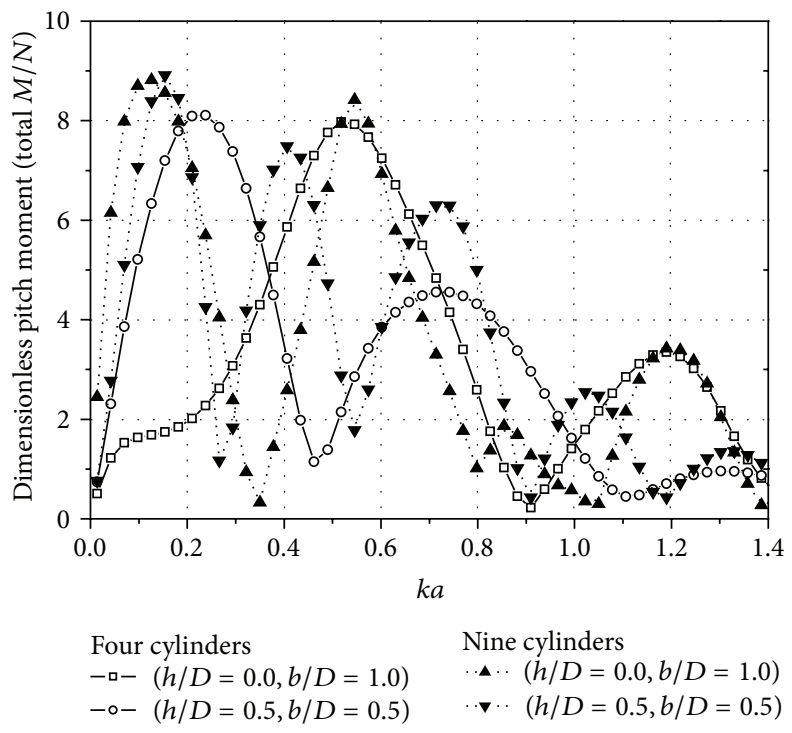

(c)

FIGURE 12: Comparison of average wave forces for four-cylinder and nine-cylinder cases with $\beta=0^{\circ}$ : (a) surge force, (b) heave force, and (c) pitch moment; other conditions are same as Figure 3.

cylinders can be used to optimize the design of structures in order to reduce the wave loads to which they are subjected.

\section{Conflict of Interests}

The authors declare that there is no conflict of interests regarding the publication of this paper.

\section{Acknowledgments}

This study was supported by Korea Institute of Marine Science and Technology Promotion through the research project "Safety evaluation of concrete substructure systems for offshore wind power (20120093)." This work was also financially supported by the Ministry of Trade, Industry and Energy (MOTIE), Korea Institute for Advancement of Technology (KIAT), and DongNam Institute for Regional Program Evaluation (IRPE) through the Leading Industry Development for Economic Region.

\section{References}

[1] B. H. Spring and P. L. Monkmeyer, "Interaction of plane waves with vertical cylinders," in Proceedings of the 14th International Conference on Coastal Engineering, vol. 107, pp. 1828-1845, 1974.

[2] C. M. Linton and D. V. Evans, "The interaction of waves with arrays of vertical circular cylinders," Journal of Fluid Mechanics, vol. 215, pp. 549-569, 1990. 
[3] H. Kagemoto and D. K. P. Yue, "Interactions among multiple three-dimensional bodies in water waves; an exact algebraic method," Journal of Fluid Mechanics, vol. 166, pp. 189-209, 1986.

[4] P. McIver and D. V. Evans, "Approximation of wave forces on cylinder arrays," Applied Ocean Research, vol. 6, no. 2, pp. 101$107,1984$.

[5] P. McIver, "Wave forces on arrays of floating bodies," Journal of Engineering Mathematics, vol. 18, no. 4, pp. 273-285, 1984.

[6] A. N. Williams and Z. Demirbilek, "Hydrodynamic interactions in floating cylinder arrays-I. Wave scattering," Ocean Engineering, vol. 15, no. 6, pp. 549-583, 1988.

[7] A. N. Williams and A. G. Abul-Azm, "Hydrodynamic interactions in floating cylinder arrays-II. Wave radiation," Ocean Engineering, vol. 16, no. 3, pp. 217-263, 1989.

[8] A. N. Williams and T. Rangappa, "Approximate hydrodynamic analysis of multi-column ocean structures," Ocean Engineering, vol. 21, no. 6, pp. 519-573, 1994.

[9] M. H. Kim, "Interaction of waves with $\mathrm{N}$ vertical circular cylinders," Journal of Waterway, Port, Coastal and Ocean Engineering, vol. 119, no. 6, pp. 671-689, 1993.

[10] O. Yilmaz, "Hydrodynamic interactions of waves with group of truncated vertical cylinders," Journal of Waterway, Port, Coastal, and Ocean Engineering, vol. 124, no. 5, pp. 272-279, 1998.

[11] P. Siddorn and R. E. Taylor, "Diffraction and independent radiation by an array of floating cylinders," Ocean Engineering, vol. 35, no. 13, pp. 1289-1303, 2008.

[12] A. N. Williams and W. Li, "Wave interaction with a semiporous cylindrical breakwater mounted on a storage tank," Ocean Engineering, vol. 25, no. 2-3, pp. 195-219, 1998.

[13] A. N. Williams and W. Li, "Water wave interaction with an array of bottom-mounted surface-piercing porous cylinders," Ocean Engineering, vol. 27, no. 8, pp. 841-866, 2000.

[14] H. I. Cho and M. H. Kim, "Wave deformation and blocking performance by $\mathrm{N}$ porous bottom-mounted vertical circular cylinders," International Journal of Offshore and Polar Engineering, vol. 20, no. 4, pp. 284-291, 2010.

[15] M. S. Park, W. C. Koo, and Y. R. Choi, "Hydrodynamic interaction with an array of porous circular cylinders," International Journal of Naval Architecture and Ocean Engineering, vol. 2, no. 3, pp. 146-154, 2010.

[16] F. Zhao, T. Kinoshita, W. Bao, and H. Itakura, "Wave fields diffracted by an array of porous circular cylinders," in Proceedings of the ASME 29th International Conference on Ocean, Offshore and Arctic Engineering (OMAE '10), pp. 65-74, Shanghai, China, June 2010.

[17] K. H. Wang and X. Ren, "Wave interaction with a concentric porous cylinder system," Ocean Engineering, vol. 21, no. 4, pp. 343-360, 1994.

[18] K. Sankarbabu, S. A. Sannasiraj, and V. Sundar, "Interaction of regular waves with a group of dual porous circular cylinders," Applied Ocean Research, vol. 29, no. 4, pp. 180-190, 2007.

[19] F. Zhao, W. Bao, T. Kinoshita, and H. Itakura, "Theoretical and experimental study on a porous cylinder floating in waves," Journal of Offshore Mechanics and Arctic Engineering, vol. 133, no. 1, Article ID 011301, 2011.

[20] M. S. Park, K. Kawano, and S. Nagata, "Numerical analysis of an offshore platform with large partial porous cylindrical members due to wave forces," Ocean Systems Engineering, vol. 1, no. 4, pp. 337-353, 2011.

[21] M. S. Park, Y. Jeong, and Y. J. You, "Numerical analysis of an offshore platform with partial porous cylinders due to wave excitation forces and seismic forces," in Oceans 2012, pp. 1-9, IEEE, Hampton Road, Va, USA, October 2012.

[22] M. S. Park, Y. J. Jeong, Y. J. You, and J. H. Park, "Reliability evaluations of an offshore platform with partial porous cylinders due to wave and seismic forces," in Proceedings of the 32nd International Conference on Ocean, Offshore and Arctic Engineering (OMAE '13), V02AT02A042, ASME, 2013.

[23] Y. R. Choi, S. Y. Hong, and H. S. Choi, "An analysis of secondorder wave forces on floating bodies by using a higher-order boundary element method," Ocean Engineering, vol. 28, no. 1, pp. 117-138, 2001.

[24] M. Abramowitz and I. A. Stegun, Handbook of Mathematical Functions, Dover, New York, NY, USA, 1972. 


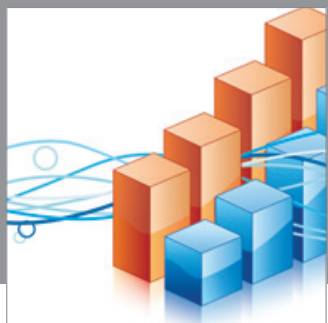

Advances in

Operations Research

mansans

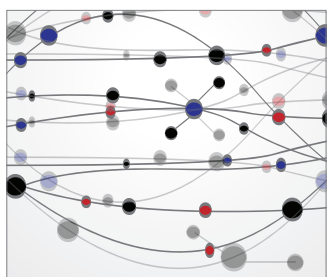

The Scientific World Journal
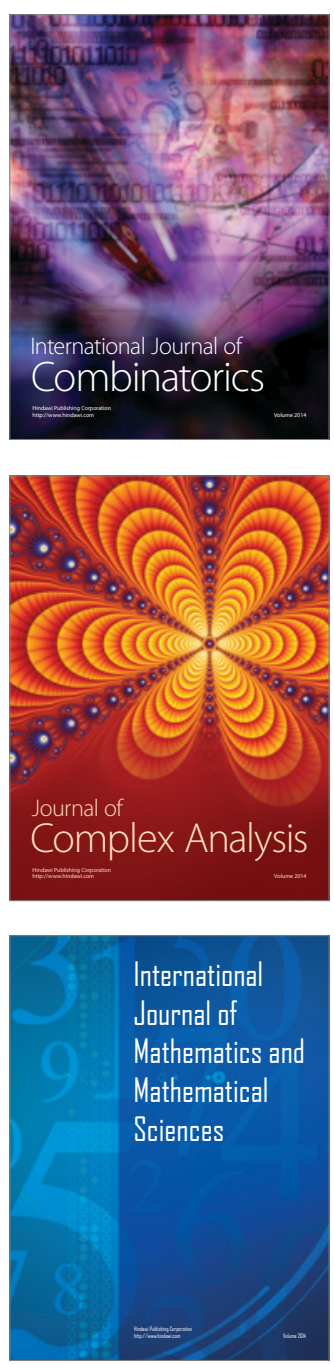
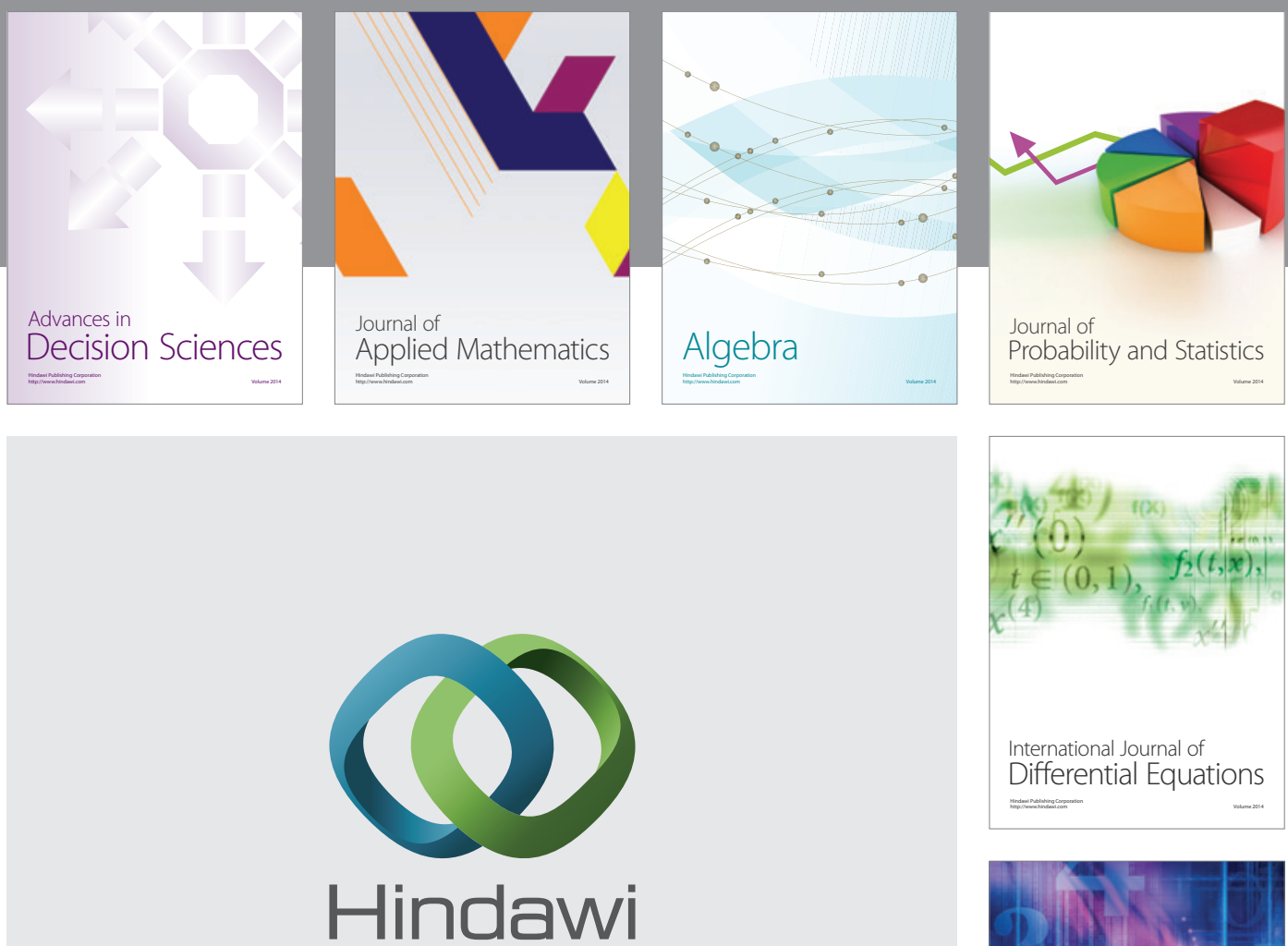

Submit your manuscripts at http://www.hindawi.com
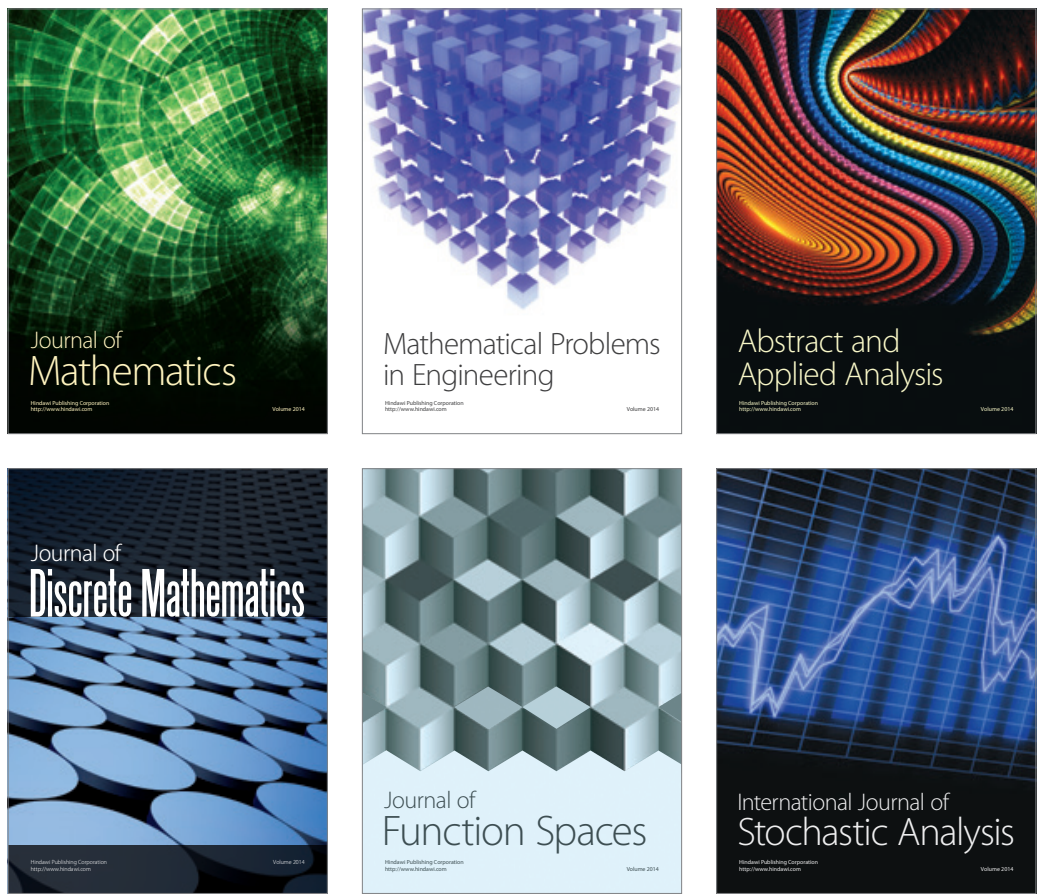

Journal of

Function Spaces

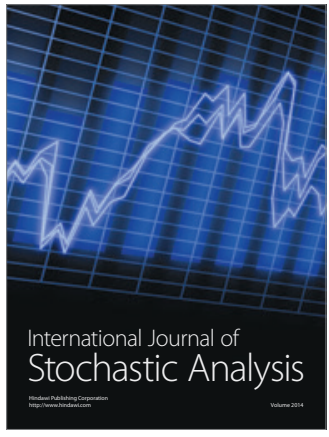

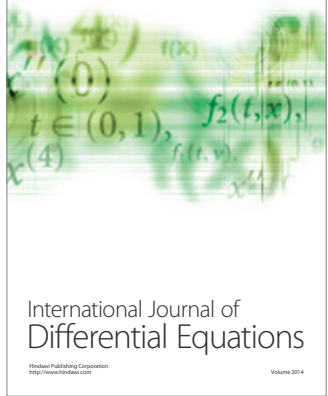
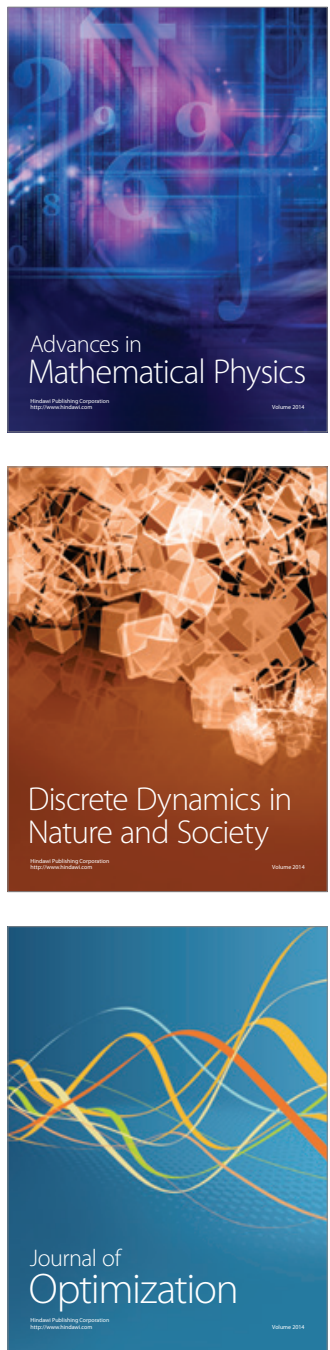